AWARD NUMBER: W81XWH-12-1-0383

TITLE: Targeting Redox Homeostasis in LKB1-deficient NSCLC

PRINCIPAL INVESTIGATOR: Jamey D. Young

CONTRACTING ORGANIZATION: The Vanderbilt University

Nashville, TN 37240

REPORT DATE: September 2014

TYPE OF REPORT: Final

PREPARED FOR: U.S. Army Medical Research and Materiel Command

Fort Detrick, Maryland 21702-5012

DISTRIBUTION STATEMENT: Approved for Public Release;

Distribution Unlimited

The views, opinions and/or findings contained in this report are those of the author(s) and should not be construed as an official Department of the Army position, policy or decision unless so designated by other documentation. 


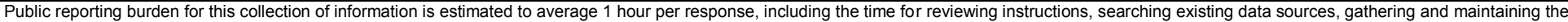

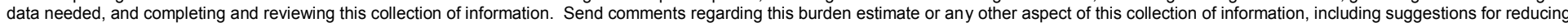

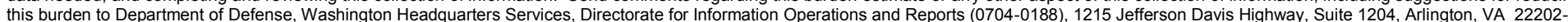

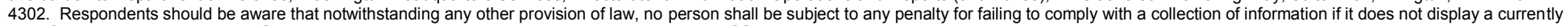
valid OMB control number. PLEASE DO NOT RETURN YOUR FORM TO THE ABOVE ADDRESS

\begin{tabular}{l|l}
\hline 1. REPORT DATE & 2. REPORT TYPE \\
Septemebr 2014 & Final \\
\hline
\end{tabular}

4. TITLE AND SUBTITLE

Targeting Redox Homeostasis in LKB1-deficient NSCLC
3. DATES COVERED

15 Sep 2012 - 14 Sep 2014

5a. CONTRACT NUMBER

5b. GRANT NUMBER

W81XWH-12-1-0383

5c. PROGRAM ELEMENT NUMBER

5d. PROJECT NUMBER

6. AUTHOR(S)

Jamey D. Young, PI

Young Mi Whang, postdoctoral associate

E-Mail: j.d.young@ vanderbilt.edu

7. PERFORMING ORGANIZATION NAME(S) AND ADDRESS(ES)

The Vanderbilt University

Nashville TN 37240-0001

9. SPONSORING / MONITORING AGENCY NAME(S) AND ADDRESS(ES)

10. SPONSOR/MONITOR'S ACRONYM(S)

U.S. Army Medical Research and Materiel Command

Fort Detrick, Maryland 21702-5012

11. SPONSOR/MONITOR'S REPORT

NUMBER(S)

\section{DISTRIBUTION / AVAILABILITY STATEMENT}

Approved for Public Release; Distribution Unlimited

\section{SUPPLEMENTARY NOTES}

\section{ABSTRACT}

LKB1 is a master kinase that is mutated or underexpressed in $20-30 \%$ of non-small cell lung cancer (NSCLC) patient tumors. Because wild-type LKB1 functions as a sensor of energetic stress, the response to metabolic inhibition or nutrient depletion becomes dysregulated in LKB1-deficient cells. We have assessed the metabolic response to treatment of LKB1-deficient NSCLC cells with the clinically relevant EGFR inhibitor erlotinib. LKB1-deficient cells exhibited enhanced sensitivity to erlotinib treatment despite having wild-type EGFR. We have found that this enhanced response is due to mitochondrial dysfunction and altered energetic metabolism in LKB1-deficient cells, which prevents homestatic maintenance of ATP and reactive oxygen species (ROS) levels in response to erlotinib treatment. This subsequently results in reductions in $\mathrm{mTOR}$ signaling and cell growth, as well as activation of apoptosis. These findings will be important for designing targeted treatments for LKB1-deficient NSCLC patient tumors, which disrupt metabolic and signaling pathways known to regulate energy metabolism in these cells.

\section{SUBJECT TERMS}

EGFR, epidermal growth factor receptor; NSCLC, non-small cell lung cancer; ROS, reactive oxygen species

\begin{tabular}{|c|c|c|c|c|c|}
\hline \multicolumn{3}{|c|}{ 16. SECURITY CLASSIFICATION OF: } & \multirow{2}{*}{$\begin{array}{l}\text { 17. LIMITATION } \\
\text { OF ABSTRACT } \\
\text { Unclassified }\end{array}$} & \multirow{2}{*}{$\begin{array}{c}\text { 18. NUMBER } \\
\text { OF PAGES } \\
36\end{array}$} & \multirow{2}{*}{$\begin{array}{l}\text { 19a. NAME OF RESPONSIBLE PERSON } \\
\text { USAMRMC } \\
\text { 19b. TELEPHONE NUMBER (include area } \\
\text { code) }\end{array}$} \\
\hline $\begin{array}{l}\text { a. REPORT } \\
\text { Unclassified }\end{array}$ & $\begin{array}{l}\text { b. ABSTRACT } \\
\text { Unclassified }\end{array}$ & $\begin{array}{l}\text { c. THIS PAGE } \\
\text { Unclassified }\end{array}$ & & & \\
\hline
\end{tabular}




\section{Table of Contents}

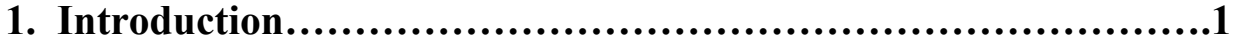

2. Keywords...........................................................1

3. Accomplishments.................................................1

4. Impact..................................................................2

5. Changes/Problems...................................................2

6. Products...............................................................2

7. Participants \& Other Collaborating Organizations..............3

8. Special Reporting Requirements...................................3

9. Appendices...........................................................4 


\section{INTRODUCTION}

The overall objective of the current application is to apply metabolic flux analysis and quantitative metabolic profiling to identify critical nodes that regulate energy metabolism and antioxidant functions in LKB1-deficient NSCLC cells. LKB1 is a tumor suppressive serine/threonine kinase that activates diverse downstream kinases, thus regulating a variety of cellular phenotypes including metabolism, invasion, proliferation, and polarity [1]. LKB1 is lost in 20-30 percent of lung adenocarcinomas and 10-20 percent of lung squamous cell carcinomas, as well as in other primary tumor types at a lower prevalence. Our central hypothesis is that LKB1-deficient NSCLC cells have increased sensitivity to treatments that interfere with energy metabolism. Our long-term goal is to understand mechanisms of metabolic reprogramming in lung tumor cells so that these processes can be targeted by molecular therapeutics.

\section{KEYWORDS}

EGFR, epidermal growth factor receptor; NSCLC, non-small cell lung cancer; ROS, reactive oxygen species

\section{ACCOMPLISHMENTS}

1. Determine the metabolic response of LKB1-deficient NSCLC cells to inhibiting mitochondrial oxidative metabolism.

Refer to draft manuscript (Appendix A) for a description of accomplishments under Aim 1.

2. Assess the roles of NADPH-producing pathways in regulating redox homeostasis of LKB1deficient NSCLC cells.

Reactive oxygen species (ROS) production correlates with Nrf2-associated gene expression signature. Gene expression studies in Dr. Carbone's lab have revealed that a major component of the LKB1-deficient transcriptional signature is comprised of Nrf2 target genes involved in ROS detoxification, including enzymes involved in NADPH production. Nrf 2 activation is observed in approximately half of tumors that have lost LKB1 function, but only $6 \%$ of LKB1 wild-type tumors. Analysis of mutation data reveals that this Nrf2-associated gene expression phenotype is driven primarily by somatic mutations in Keap1, of which $80 \%$ occur in tumors with LKB1 loss. LKB1-deficient tumors have been shown to be susceptible to oxidative stress, as they are unable to make the appropriate adaptive responses in metabolism and biosynthesis [2]. Thus, the high frequency of Nrf2 activation among LKB1-deficient tumors suggests that selective pressure exists for these mutations as a secondary protective mechanism. We have profiled endogenous ROS production in several LKB1 wild-type and mutant cell lines, which revealed significantly lower baseline ROS levels in LKB1-mutant NSCLC cells (Fig. 1). Taken together, these results indicate that Nrf2-activating mutations are closely associated with LKB1 deficiency and that Nrf2 activation functions to detoxify endogenous ROS in NSCLC cells.

LKB1 loss enhances Nrf2 activation in cells with wild-type KEAP1. We asked whether some of the metabolic alterations associated with LKB1 deficiency could be caused by alterations in Nrf2 activation. Experiments with Calu-6 cell lines transfected with shLKB1 either transiently or stably show enhanced transcription from Nrf2 target genes (Fig. 2A). Furthermore, increased phosphorylation and nuclear translocation of Nrf2 was observed after LKB1 knockdown (Fig. 
2B). Although stable LKB1-knockdown Calu-6 cells showed both increased Nrf2

phosphorylation and expression, overexpression of LKB1 in mutant lines A549 and H2122 did not produce the reverse effect. We hypothesize that this is due to the presence of KEAP1 mutations in both A549 and $\mathrm{H} 2122$ that stabilize Nrf2, thus altering the response to LKB1 overexpression.

\section{IMPACT}

Major findings of this research to date:

- LKB1-deficient cells are more sensitive to inhibition of EGFR-PI3K-mTOR signaling.

- Erlotinib treatment induces energetic stress and ROS accumulation in LKB1-deficient cells.

- The enhanced susceptibility of LKB1-deficient cells is associated with defective mitochondrial metabolism.

- AMPK $\alpha$ activation by erlotinib blocks mTOR signaling in LKB1-deficient cells.

- ROS production correlates with Nrf2-associated gene expression signature in LKB1deficient cells.

- Nrf2 is upregulated following transient or stable knockdown of LKB1 in Calu-6 cells.

- Overexpressing LKB1 in cell lines with KEAP1 mutations does not reduce Nrf2 activation.

\section{CHANGES/PROBLEMS}

N/A

\section{PRODUCTS}

Manuscript: Y. M. Whang, S. I. Park, I. A. Trenary, R. A. Egnatchik, J. Kaufman, D. P. Carbone, J. D. Young*. LKB1 deficiency enhances sensitivity to energetic stress induced by erlotinib treatment in non-small cell lung cancer (NSCLC) cells. Oncogene, submitted.

Manuscript in preparation: C. Lee, Y. M. Whang, P. Campbell, P. Mulcrone, J. D. Young, F. Elefteriou, S. I. Park*. Dual targeting c-Met and VEGFR2 in the stromal cells suppresses growth and osteolysis of prostate cancer bone metastasis.

Conference presentation: Y. M. Whang*, S. I. Park, I. A Trenary, C. Lee, J. Kaufman, D. P. Carbone, J. D. Young. LKB1deficiency enhances sensitivity to energetic stress induced by erlotinib treatment in non-small cell lung cancer (NSCLC) cells. Poster 1839, AACR Annual Meeting 2014, San Diego, CA, April 5-9, 2014.

Conference presentation: C. Lee, P. Campbell, Y. M. Whang, J. D. Young, F. Elefteriou, S. I. Park*. Targeting c-Met and VEGFR2 in the stromal compartment of prostate cancer bone metastasis. Poster 1172, AACR Annual Meeting 2014, San Diego, CA, April 5-9, 2014.

Invited talk: J. D. Young. Methods Workshop: Approaches and Challenges in Studying Cancer Metabolism. AACR Annual Meeting 2014, San Diego, CA. Apr 5, 2014.

Invited talk: Uniting Against Lung Cancer, Investigator Meeting. New York, NY. Nov 15, 2012. 
Cell lines: shLKB1 knockdown derivatives of Calu-6 and H358, stable LKB1-overexpressing derivatives of A549, $\mathrm{H} 2122$, and $\mathrm{H} 460$.

Training support for postdoctoral researcher, Dr. Young-Mi Whang.

\section{PARTICIPANTS AND OTHER COLLABORATING ORGANIZATIONS}

Vanderbilt University

Jamey D. Young, PI

Young Mi Whang, postdoctoral scholar

Irina Trenary, research assistant

Robert Egnatchik, graduate student

Jacob Kaufman, medical student

Serk In Park, collaborator

Ohio State University

David Carbone, Co-PI

\section{SPECIAL REPORTING REQUIREMENTS}

N/A

\section{REFERENCES}

1. Shackelford DB, Shaw RJ (2009) The LKB1-AMPK pathway: metabolism and growth control in tumour suppression. Nat Rev Cancer 9: 563-575.

2. Jeon SM, Chandel NS, Hay N (2012) AMPK regulates NADPH homeostasis to promote tumour cell survival during energy stress. Nature 485: 661-665. 


\section{APPENDIX A}

Supporting data.

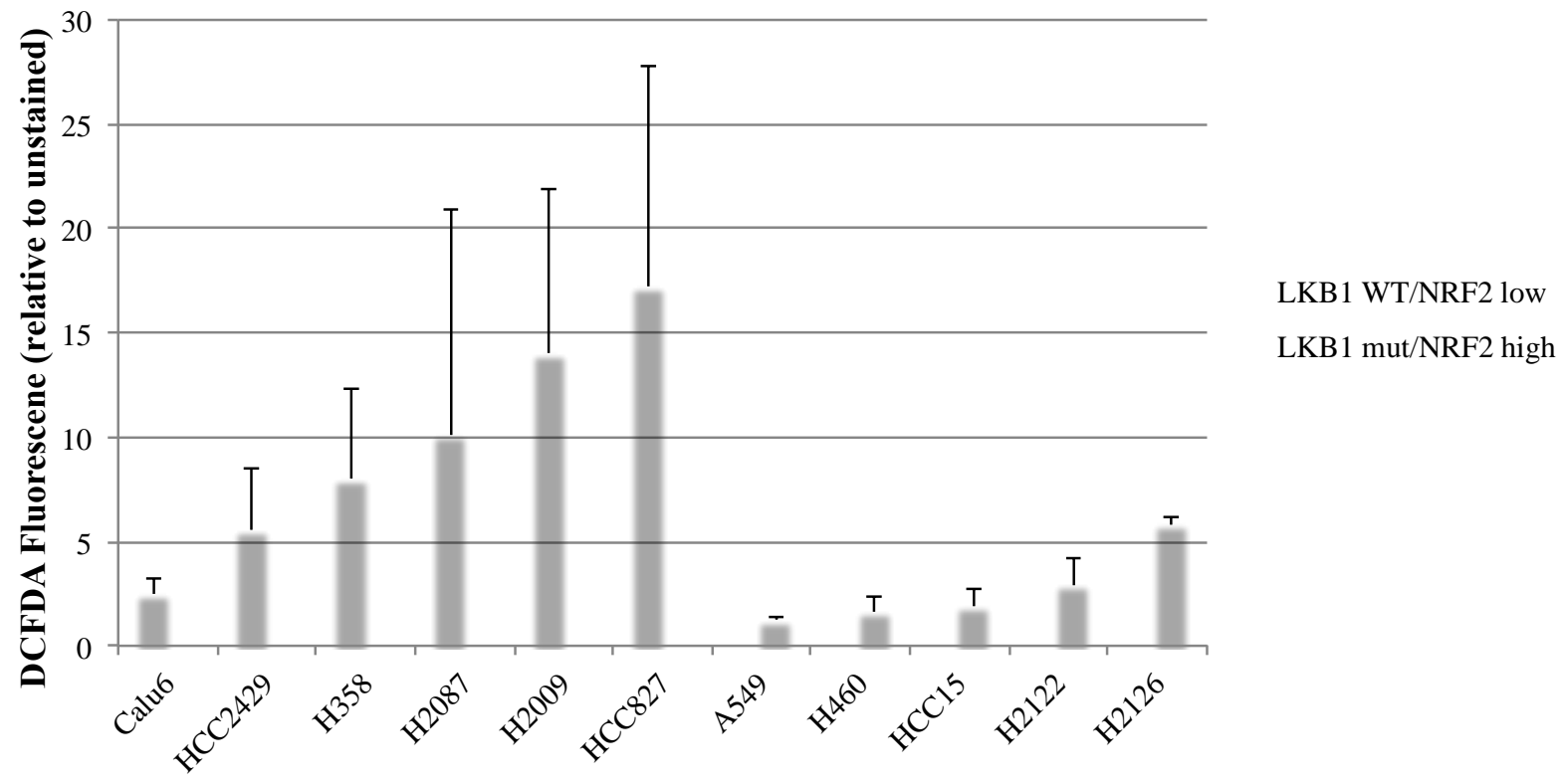

Figure 1. Endogenous ROS production among NSCLC cell lines. The activation of NRF2, as determined by gene expression profiling, was associated with significantly lower levels of endogenous ROS in cell lines. A549 and H460 have known KEAP1 mutations. 

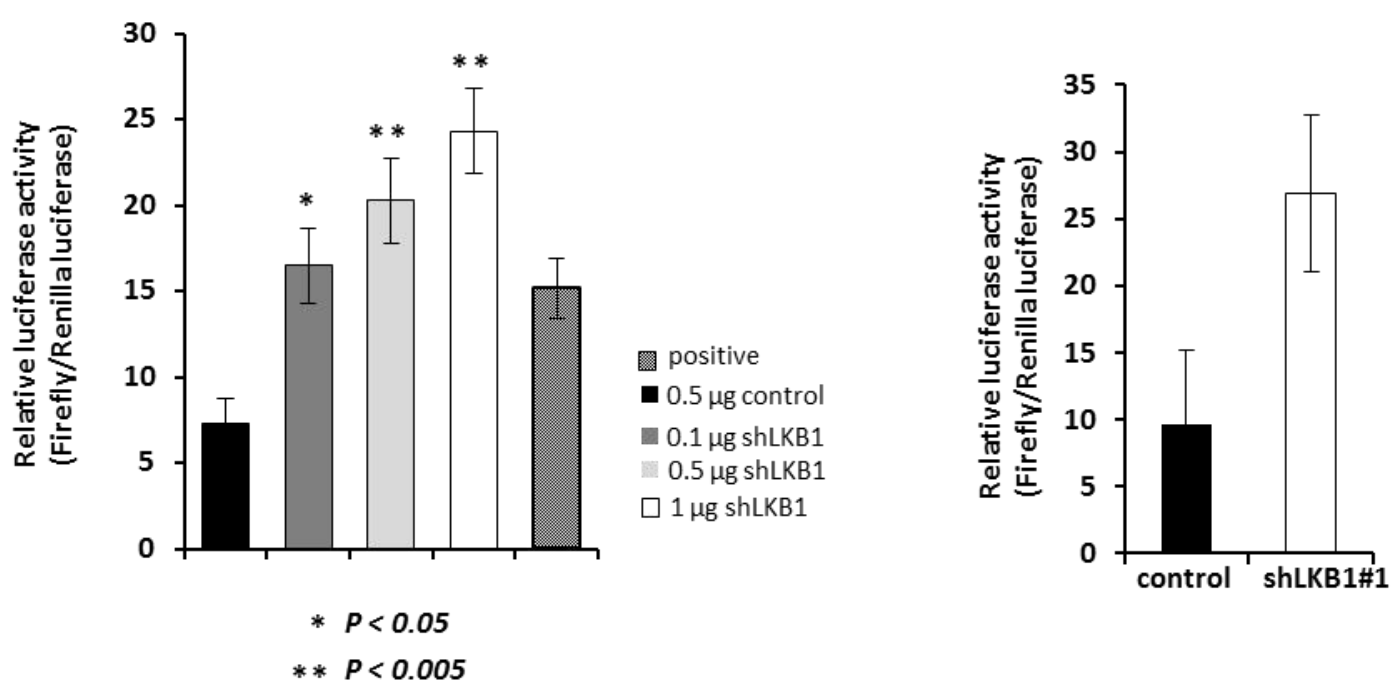

B

C

Calu-6
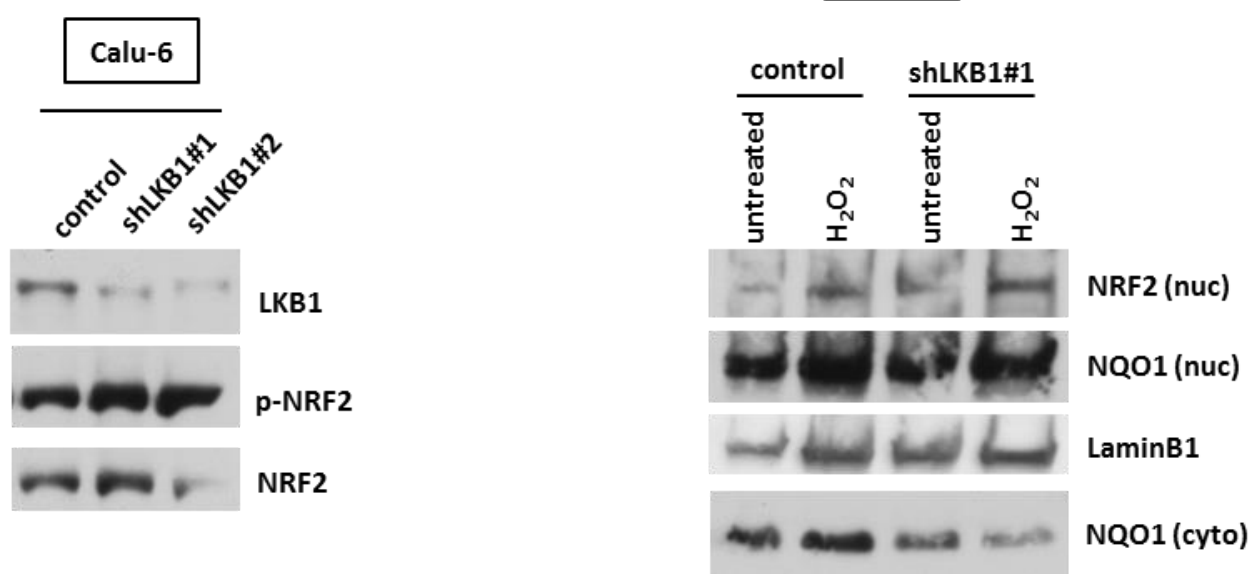

Figure 2. LKB1 loss is associated with NRF2 activation. A, LKB1 depletion induces activation of NRF2-dependent transcription. Left panel: Calu-6 cells were transiently cotransfected with ARE-luciferase plasmid and shRNA constructs of LKB1 at indicated shRNA concentrations. Right panel: stable knockdown LKB1 and control Calu-6 were transfected with ARE-luciferase plasmid. After $48 \mathrm{~h}$ post-transfection, the cell lysates were subjected to dual luciferase assay transfection. The signals were normalized by corresponding renilla signals, by firefly/renilla ratio. B, LKB1 depletion induces phosphorylation of NRF2. After $48 \mathrm{~h}$ posttransfection, transient LKB1 knockdown Calu- 6 cells were analyzed with the indicated antibodies by Western blotting. C, Localization of NRF2 by treatment with $\mathrm{H}_{2} \mathrm{O}_{2}$ in LKB1 stable knockdown Calu- 6 cells. Cells were stimulated with $100 \mu \mathrm{M} \mathrm{H}_{2} \mathrm{O}_{2}$ for $4 \mathrm{~h}$ and nuclear extracts were analyzed by western blotting. 

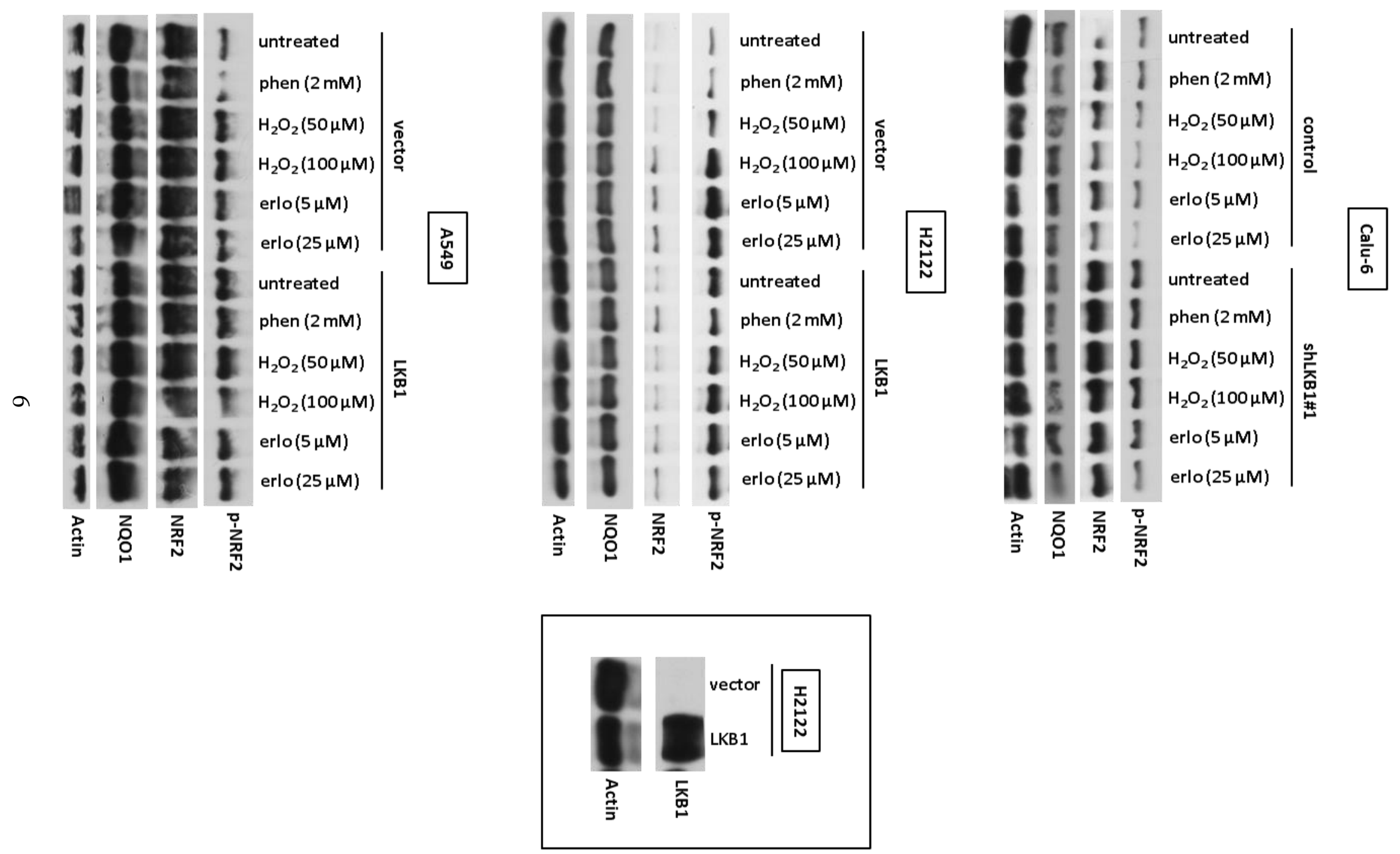
Figure 3. NRF2 activation is upregulated by LKB1 loss but does not respond to restoration of LKB1 in KEAP1-mutant lines. A, stable knockdown Calu-6 cells exhibited high levels of NRF2 expression. Cells were treated with indicated agents for $24 \mathrm{~h}$ and the cell lysates were analyzed with the indicated antibodies by Western blotting. B and C, H2122 cells (LKB1 MT/KEAP1 MT) and A549 (LKB1 MT/KEAP1 MT) stably overexpressing LKB1were treated with indicated agents for $4 \mathrm{~h}$ and the cell lysates were then analyzed by Western blotting. Box: Levels of LKB1 expression in $\mathrm{H} 2122$ cells stably expressing empty pBABE vector (vector) or wild-type LKB1 (LKB1). 


\section{APPENDIX B}

Draft manuscript submitted to Oncogene. 


\title{
LKB1 deficiency enhances sensitivity to energetic stress induced by erlotinib treatment in non- small cell lung cancer (NSCLC) cells
}

Young Mi Whang ${ }^{1}$, Serk In Park ${ }^{4}$, Irina A. Trenary ${ }^{1}$, Robert A. Egnatchik ${ }^{1}$, Jacob M. Kaufman ${ }^{3}$, David P. Carbone ${ }^{5}$, and Jamey D. Young ${ }^{1,2, *}$

${ }^{1}$ Department of Chemical and Biomolecular Engineering, ${ }^{2}$ Department of Molecular Physiology and Biophysics, ${ }^{3}$ Department of Cancer Biology, Vanderbilt University; ${ }^{4}$ Departments of Clinical Pharmacology Division/Medicine, Vanderbilt University School of Medicine; ${ }^{5}$ Department of Internal Medicine, The Ohio State University Medical Center

* Correspondence to: Jamey D. Young, Phone: 615-343-4253, Fax: 615-343-7951, E-mail: j.d.young@vanderbilt.edu

The authors declare no conflict of interest.

\begin{abstract}
The tumor suppressor serine/threonine kinase 11 (STK11 or LKB1) is mutated in 20-30\% of non-small cell lung cancer (NSCLC) patient tumors. Loss of LKB1-AMPK signaling confers sensitivity to metabolic inhibition or stress-induced mitochondrial insults. We tested the hypothesis that loss of LKB1 sensitizes NSCLC cells to energetic stress induced by treatment with erlotinib. LKB1-deficient cells exhibited enhanced sensitivity to erlotinib in vitro and in vivo that was associated with alterations in energy metabolism and mitochondrial dysfunction. Knockdown of LKB1 altered the cellular response to erlotinib treatment, resulting in impaired ATP homeostasis and an increase in reactive oxygen species (ROS). Furthermore, erlotinib selectively blocked mTOR signaling, inhibited cell growth, and activated apoptosis in LKB1-deficient cells. Erlotinib treatment also induced AMPK activation despite knockdown of LKB1, which was attenuated by the application of a CaMKK $\beta$ inhibitor (STO-609) and calcium chelator (BAPTA-AM). These findings may have significant implications for the design of novel NSCLC treatments that target dysregulated metabolic and signaling pathways in LKB1-deficient tumors.
\end{abstract}

\section{Introduction}

Lung cancer is the leading cause of cancer mortality worldwide and is linked to $28 \%$ of all cancer deaths in the United States (1). Despite advances in traditional therapeutic strategies involving surgery, ionizing radiation therapy, and chemotherapy, the 5-year survival remains less than $20 \%$ (2). 
In recent years, it has become clear that non-small cell lung cancer has a high frequency of somatically acquired genetic alterations that define critical subsets of tumors with distinct behaviors (3). An improved understanding of potential vulnerabilities of lung cancer subsets has led to the development of effective targeted therapies for tumors with certain activated oncogenes $(4,5)$, but little is known about specific susceptibilities that may derive from the loss of classical tumor suppressor genes, such as $L K B 1$.

LKB1 is a tumor suppressive serine/threonine kinase that activates diverse downstream kinases, thus regulating a variety of cellular phenotypes including metabolism, invasion, proliferation, and polarity (6). $L K B 1$ inactivation occurs in 20-30 percent of lung adenocarcinomas (7), and $L K B 1$ deficiency in combination with $K R A S$ mutation leads to an aggressive tumor phenotype at high prevalence in mouse models, surpassing that of KRAS mutation alone (8). Many of the metabolic regulatory functions of LKB1 are mediated by its interaction with adenosine monophosphateactivated protein kinase (AMPK). LKB1 phosphorylates and activates AMPK (6), which functions to regulate cellular energy metabolism under conditions of low ATP (9). AMPK also contributes to inactivation of mTOR when ATP levels fall, which leads to inhibition of protein synthesis and cell growth (10). Therefore, loss of LKB1 leads to dysregulation of cellular metabolism and cell growth under conditions of energy stress (11), resulting in enhanced sensitivity to drug treatments that target bioenergetic pathways (12).

Most lung cancers express the epidermal growth factor receptor (EGFR), and this signaling pathway is the major target of several drugs in the clinic. EGFR tyrosine kinase inhibitors including gefitinib and erlotinib have been shown to suppress oncogenic signaling through downstream pathways such as PI3K-Akt-mTOR and Mek-Erk (13). NSCLC tumors with certain activating mutations in $E G F R$ show enhanced sensitivity to these compounds (14). However, the majority of NSCLC patient tumors possess wild-type EGFR, with only $10-30 \%$ harboring a mutant $E G F R$ allele (15). Although erlotinib has clear therapeutic efficacy in some NSCLC tumors bearing wild-type $\operatorname{EGFR}(16,17)$ and is approved for patients with wild-type EGFR tumors $(18)$, it is unclear how to best identify which of these patients may benefit from treatment with EGFR-targeted inhibitors. Furthermore, the mechanism by which erlotinib induces selective cell death in wild-type EGFR tumors is not completely known.

In EGFR mutant NSCLC cells, erlotinib causes apoptosis through activation of intrinsic pathways mediated by the induction of BH3-only BIM protein or activation of caspase $3(19,20)$. In these studies, erlotinib treatment was associated with loss of mitochondrial potential, which resulted in mitochondrial-mediated apoptosis. Interestingly, recent studies suggest that LKB1 deficiency causes an accumulation of defective mitochondria and loss of mitochondrial membrane potential, resulting in depletion of hematopoietic stem cells through disruption of mitophagy and mitochondrial 
homeostasis (21). Furthermore, the mitochondrial complex I inhibitor phenformin enhanced apoptosis of LKB1-deficient tumor cells by depletion of mitochondrial membrane potential compared to wildtype LKB1-reconstituted cells (12). Therefore, we hypothesized that erlotinib would be more effective at inducing apoptosis in LKB1-deficient NSCLC cells due to disruption of normal mitochondrial function, even in the presence of wild-type EGFR.

In the present study, we tested whether LKB1-deficient cells were more prone to apoptosis in response to erlotinib treatment, both in vitro and in vivo, in the absence of mutationally activated EGFR. Our data demonstrate enhanced antitumor effects of erlotinib in LKB1-deficient cells that are associated with loss of mitochondrial function and blockage of mTOR signaling. Overall, our findings suggest that the LKB1-deficient molecular phenotype may serve as a predictive marker for erlotinib sensitivity and, if substantiated by further studies, may provide a rationale for the therapeutic use of erlotinib to treat LKB1-deficient NSCLC. This is significant because LKB1 mutations are preferentially found in NSCLC tumors with wild-type EGFR $(22,23)$, and thus LKB1 loss may define a novel subset of tumors with enhanced erlotinib sensitivity that is largely distinct from the classical subset possessing targetable EGFR mutations.

\section{Materials and Methods}

\section{Cell culture and reagents}

Human NSCLC Calu-6, H358, H2009, H460, H23, H2122, HCC15, and A549 cell lines were generously shared with us by John Minna and Luc Girard (University of Texas, Southwestern). Cells were tested to ensure that they were mycoplasma negative. The human NSCLC cells were maintained in RPMI 1640 medium supplemented with 10\% fetal bovine serum (FBS; GIBCO-BRL, Gaithersburg, MD) in a humidified atmosphere with $5 \% \mathrm{CO}_{2}$. A549 and $\mathrm{H} 460$ cells stably expressing either an empty pBABE vector (vector) or wild-type LKB1 (LKB1) were generated by retroviral transduction. The empty pBABE viral plasmid and pBABE-LKB1 plasmid were obtained from Addgene (Cambridge, MA). Phoenix cells were transfected with viral plasmids and retroviral particles were harvested from media supernatant 48 hours after transfection. Viruses were added to target cells with polybrene, and selection with $1 \mu \mathrm{g} / \mathrm{ml}$ puromycin was begun 48-72 hours after infection. Cells were selected under puromycin for one to two weeks before performing subsequent experiments, with experiments being completed within two months. Erlotinib (an inhibitor of EGFR), LY294002 (an inhibitor of PI3K), and rapamycin (an inhibitor of mTOR) were purchased from LC Laboratories (Woburn, MA).

Cell viability analysis, colony formation assays, and growth rate measurements 
Cells were plated in 96-well plates in complete medium and treated with various concentrations of erlotinib, LY294002, or rapamycin. Cell viability was analyzed after 2 days of treatment using the 3-(4,5-dimethylthiazol-2-yl)-2,5-diphenyltetrazolium bromide (MTT) assay according to the manufacturer's instructions (Sigma, St. Louis, MO). Cell proliferation was analyzed every 8 or $16 \mathrm{~h}$ for 3 days after erlotinib treatment using the CyQUANT ${ }^{\circledR}$ Direct Cell Proliferation Assay according to the manufacturer's protocol (Invitrogen, Frederick, MD). The fluorescence intensity was measured with a fluorescence microplate reader (Bio-Tek Instruments, Winooski, VT). Conditioned medium samples were collected from each well prior to CyQUANT analysis, frozen, and stored for metabolite analysis. Colony formation assays were performed following treatment with 5 $\mu \mathrm{M}$ erlotinib for 3 days, at which time the cells were replated in six-well plates at low density $\left(1 \times 10^{3}\right.$ cells per well) in complete medium for 2 weeks. Colonies were fixed with methanol and stained with $0.1 \%$ crystal violet (Sigma-Aldrich, St. Louis, MO). Colony numbers were assessed visually and colonies measuring at least $50 \mu \mathrm{m}$ were counted.

\section{Metabolite analysis and oxygen consumption rate}

Conditioned medium samples collected during cell growth experiments were subjected to glucose and lactate analysis using a YSI 2300 biochemical analyzer (YSI Life Science, Yellow Springs, OH). The ETA software package was applied to calculate specific rates of glucose consumption and lactate production using an exponential growth model (24). Oxygen consumption rate (OCR) was measured using the Oxygraph-2K (Oroboros, Innsbruck, Austria), which contains two chambers with separate oxygen probes to monitor on-line changes in oxygen concentration. The instrument was set to a temperature of $37^{\circ} \mathrm{C}$, and the stirring speed for each chamber was $750 \mathrm{rpm}$. After treatment with erlotinib for $24 \mathrm{~h}$, cells were then trypsinized, resuspended in the same culture medium at a concentration of $1-2 \times 10^{6}$ cells per $\mathrm{mL}$, and injected into the Oxygraph instrument.

\section{LKB1 stable knockdown using lentiviral short-hairpin RNA}

For generation of stable knockdown cells, lentiviral LKB1 short-hairpin RNA (shRNA) constructs and a non-targeting shRNA control were purchased from Open Biosystems (Huntsville, AL). These constructs were cloned into a pLKO.1 vector. HEK293T cells $\left(2 \times 10^{6}\right.$ cells $)$ were plated on $100 \mathrm{~mm}$ dishes and one day later were co-transfected with $5 \mu \mathrm{g}$ shRNA constructs, $3 \mu \mathrm{g}$ pCMVdR8.2 dvpr (Addgene) and $2 \mu \mathrm{g}$ pCMV-VSV-G (Addgene) using a calcium phosphate transfection kit (Invitrogen). After $6 \mathrm{~h}$, the medium was exchanged with fresh medium, and then the viral stock was collected for 1-2 days and filtered to remove non-adherent HEK293T cells. To obtain stable shRNA expressing clones, Calu-6 or H358 cells were plated on six-well plates, and one day later infected with $1 \mathrm{ml}$ of lentiviral suspension plus $4 \mu \mathrm{g} / \mathrm{ml}$ polybrene. Puromycin selection $(0.5-2 \mu \mathrm{g} / \mathrm{ml})$ was initiated 2 days after lentiviral infection. 


\section{Western blotting}

Total cell lysates were resolved using sodium dodecyl sulfate (SDS)-polyacrylamide gel electrophoresis, and then transferred onto polyvinylidene fluoride (PVDF) membranes (EMD Millipore Corporation, Billerica, MA). Western blotting was performed as described previously (25). The following antibodies were purchased from Cell Signaling (Danvers, MA): rabbit polyclonal antibodies against LKB1, phosphorylated AMPK $\alpha$, phosphorylated acetyl-CoA carboxylase (ACC), phosphorylated Tuberin/TSC2 (Ser1387), phosphorylated mTOR (Ser2448), phosphorylated 4EBP1 (Thr37/46), phosphorylated Akt (Ser473), phosphorylated Erk (Thr202/Tyr204), poly(ADP-ribose) polymerase (PARP), and mouse monoclonal antibodies against phosphorylated p70S6K (Thr389). A goat polyclonal anti-actin antibody was purchased from Santa Cruz (Santa Cruz, CA). All experiments were performed in triplicate.

\section{Flow cytometry}

Cells were seeded in six-well plates containing complete medium and treated with $5 \mu \mathrm{M}$ or 10 $\mu \mathrm{M}$ erlotinib. After $72 \mathrm{~h}$, cells were harvested, pooled, and fixed with $1 \%$ paraformaldehyde and $70 \%$ ethanol. Apoptosis was assessed with flow cytometry using the Annexin V-FITC Apoptosis kit (Clontech, Mountain View, CA). To measure mitochondrial ROS after $24 \mathrm{~h}$ of erlotinib treatment, cells were stained with MitoSOX Red (Molecular Probes, Eugene, OR) for $40 \mathrm{~min}$ at $37{ }^{\circ} \mathrm{C}$ in the dark. Annexin V and MitoSOX Red were analyzed using a LSRII flow cytometer (Becton Dickinson, San Jose, CA).

\section{Assessment of ADP/ATP ratio and mitochondrial membrane potential}

Cells were seeded in 96-well plates and treated with $25 \mu \mathrm{M}$ erlotinib for $24 \mathrm{~h}$. Intracellular ADP/ATP ratio was measured using a bioluminescent ADP/ATP Ratio Assay Kit according to the manufacturer's instructions (Abcam, Cambridge, MA). Mitochondrial membrane depolarization was determined using the JC-1 fluorescence probe according to the manufacturer's instructions (Molecular Probes). Cells were labeled with $2 \mu \mathrm{M} \mathrm{JC}-1$ for $30 \mathrm{~min}$ at $37{ }^{\circ} \mathrm{C}$ in the dark and then analyzed at 488 nm excitation with 530/30 or 585/42 nm bypass emission filters using a fluorescence microplate reader (Bio-TeK Instruments).

\section{Animal experiments}

The research protocol for animal studies was approved by and carried out in accordance with the Vanderbilt Institutional Animal Care \& Use Committee. Four- to five-week old female Foxn $1^{\text {nu/nu }}$ nude mice were purchased from Harlan Laboratories (Indianapolis, IN). Both non-targeting shRNA (shCon, LKB1 wild-type) and LKB1 shRNA (shLKB1, LKB1 knockdown) Calu-6 cells were injected s.c. into the left /right flanks at $5 \times 10^{6}$ cells per mouse mixed 1:1 with $100 \%$ growth factor-reduced 
Matrigel (BD Bioscience, San Jose, CA). The mice were randomly assigned to shCon + vehicle solution (in sodium carboxymethylcellulose and Tween 80), shCon + erlotinib, shLKB1+ vehicle solution, and shLKB1 + erlotinib, with each group containing eight to ten mice. After 1 week, mice were treated with vehicle or erlotinib $(100 \mathrm{mg} / \mathrm{kg})$ daily by oral gavage for 3 weeks. Tumor size was calculated by measuring the tumors in three dimensions using calipers twice a week (26), while body weight was measured twice weekly. On day 21 , mice were euthanized and tumor tissues were harvested for pathologic examination (immunohistochemical analysis) and analyzed by Western blotting.

\section{Statistical analyses}

The data acquired with MTT, colony formation, and ADP/ATP assays were analyzed using the Student's t-test. The Mann-Whitney U test was used to compare differences in tumor weight. In all of the statistical analyses, two-sided p-values less than 0.05 were considered statistically significant.

\section{Results}

\section{LKB1-deficient cells are more sensitive to inhibition of EGFR-PI3K-mTOR signaling}

We sought to determine the relative inhibitory effects of erlotinib, as well as other compounds that target the EGFR-PI3K-mTOR signaling pathway, across a panel of NSCLC cell lines that express either wild-type or mutant LKB1. The expressions of LKB1and EGFR were assessed by Western blotting in NSCLC cells (Fig. 1A). The NSCLC cells tested have been reported to express wild-type EGFR $(27,28)$, and the expression level of phosphorylated EGFR is shown in Fig 1A. Calu-6, H2009, and H358 cells were previously reported to express wild-type LKB1 (29-31), and we confirmed LKB1 protein expression in these three lines. Next, we asked whether erlotinib could enhance growth inhibition in LKB1 mutant cells harboring KRAS mutations but with wild-type $E G F R$. We found that LKB1-deficent cells were more sensitive to erlotinib on average (Fig. 1B). Furthermore, $10 \mu \mathrm{M}$ of the PI3K inhibitor LY294002 did not reduce growth in LKB1 wild-type NSCLC cells, whereas 30-50\% growth inhibition was observed in LKB1-deficient cells. Growth inhibition by rapamycin was also exacerbated in LKB1-deficient cells. Similar decreases in colony-forming ability were observed in LKB1 mutant cells after treatment with $5 \mu \mathrm{M}$ erlotinib, $10 \mu \mathrm{M}$ LY294002, or $1 \mu \mathrm{M}$ rapamycin (Fig. 1C). These results suggest that LKB1 loss confers enhanced sensitivity to inhibition of the EGFRPI3K-mTOR signaling pathway in NSCLC cells harboring wild-type EGFR.

\section{LKB1-deficient transgenic cells exhibit increased sensitivity to erlotinib treatment}


A549 and H460 cells (both wild-type EGFR and mutant $L K B 1$ ) were transduced with retroviral constructs to stably express wild-type LKB1 or an empty vector control. Expression of LKB1 and related downstream signaling proteins were verified in these stable transgenic cells (Fig. 2A, left). LKB1 phosphorylates Thr172 in the activation loop of AMPK $(32,33)$ and activates AMPK under elevated AMP levels (34). Activated AMPK subsequently phosphorylates and inhibits acetylCoA carboxylase (ACC). Overexpression of LKB1in A549 and H460 resulted in increased basal phosphorylation of AMPK $\alpha$ and its downstream target ACC. Erlotinib inhibited growth of LKB1 nonexpressing A549 $(\mathrm{p}<0.0001)$ and H460 cells $(\mathrm{p}<0.005)$ more than LKB1 overexpressing cells (Fig. 2A, right). Conversely, in stable LKB1-knockdown lines generated from LKB1 wild-type Calu- 6 cells, erlotinib induced a more substantial reduction in growth than the vector control (Fig. 2B, lower panel). Additionally, erlotinib reduced the colony-forming ability of LKB1-knockdown cells more significantly than the vector control (Fig. 2C). These results suggest that LKB1 deficiency enhances sensitivity to erlotinib by suppressing viability and tumorigenic potential of NSCLC cells.

\section{Erlotinib treatment selectively inhibits oxidative metabolism and disrupts energy homeostasis in}

\section{LKB1-deficient cells}

We next determined whether erlotinib treatment alters cellular metabolism through analysis of glucose, lactate, and oxygen exchange rates. Knockdown of LKB1 in Calu-6 cells caused basal glucose consumption and lactate production to increase significantly compared to vector control cells (Fig. 3A), consistent with previously published results (35). Measurement of oxygen consumption rate (OCR) also revealed a two-fold increase in basal oxidative metabolism by shLKB1 Calu- 6 cells. Erlotinib treatment reduced specific growth rates of both vector control and shLKB1 cells in a dosedependent manner without affecting glucose uptake or lactate production. However, the OCR of shLKB1 cells was halved by erlotinib treatment (Fig. 3B). This reduction in OCR was associated with a significant increase in the ADP/ATP ratio (Fig. 3C). In contrast, the OCR and ADP/ATP ratio of control Calu-6 cells was not affected by erlotinib. These results indicate that LKB1-deficient cells were not able to maintain their elevated rate of OXPHOS in the presence of erlotinib treatment, thus leading to ATP depletion. Several recent articles have shown that loss of LKB1 function leads to increased expression of mitochondria-associated genes (23) but also induces defective mitophagy and accumulation of dysfunctional mitochondria $(12,21)$. Therefore, we hypothesized that mitochondrial impairments may play a role in the response of LKB1-deficient cells to erlotinib treatment.

\section{Erlotinib treatment unmasks mitochondrial defects in LKB1-deficient cells}

Erlotinib-induced apoptosis is associated with loss of mitochondrial membrane potential in EGFR mutant NSCLC cells (20). Therefore, we asked whether EGFR wild-type NSCLC cells bearing loss-of-function mutations in LKB1 would show similar mitochondrial defects following erlotinib treatment. We measured the effect of erlotinib treatment on mitochondrial potential by using the 
cationic dye JC-1. Phenformin, a mitochondrial complex I antagonist, was applied as a positive control to impair mitochondria. As shown in Fig. 4A, following treatment with erlotinib or phenformin, H358 and Calu-6 cells expressing shLKB1 exhibited enhanced dissipation of $\Delta \psi_{\mathrm{m}}$ compared to control cells (red to green ratio of JC-1), reflecting impaired mitochondrial membrane integrity. Significantly, the $\Delta \psi_{\mathrm{m}}$ of untreated shLKB1 cells was higher than vector controls, which is indicative of increased basal mitochondrial activity and is consistent with the elevated OCR of untreated shLKB1 Calu-6 cells (Fig. 3B). Calu-6 cells expressing shLKB1 also exhibited increased mitochondrial production of reactive oxygen species (ROS) in response to erlotinib treatment (Fig. 4B). We hypothesized that ATP depletion, loss of mitochondrial membrane potential, and ROS accumulation might by associated with increased apoptosis in LKB1-deficient cells. As shown in Fig. 4C and 4D, treatment of shLKB1 Calu-6 cells with erlotinib induced cleavage of PARP and an accumulation of apoptotic cells, suggesting that erlotinib enhances mitochondrial-mediated apoptosis in LKB1-deficient cells. These data indicate that shLKB1 Calu-6 cells rely more heavily on mitochondria for energy metabolism under basal conditions, but they exhibit profound mitochondrial dysfunction in response to erlotinib treatment as evidenced by ATP depletion, ROS accumulation, and apoptosis activation.

\section{Erlotinib activates AMPKa and blocks mTOR signaling in LKB1-deficient cells}

Next, we evaluated the effect of erlotinib on phosphorylation of AMPK $\alpha$ at 3, 6, and $24 \mathrm{~h}$ post-treatment. Interestingly, erlotinib induced phosphorylation of AMPK $\alpha$ after $6 \mathrm{~h}$ treatment in shLKB1 Calu-6 cells (Fig. 5A, left panel), with increased phosphorylation of acetyl-CoA carboxylase (ACC) apparent by $24 \mathrm{~h}$. This effect was not observed in the control cells. In non-transgenic LKB1 mutant NSCLC cells, erlotinib also induced phosphorylated AMPK $\alpha$ in both H23 and H460 cells after $3 \mathrm{~h}$ treatment (Fig. 5A, right panel). These results indicate that AMPK $\alpha$ remains sensitive to changes in ATP levels induced by erlotinib treatments in spite of reduced or abolished LKB1 activity. To confirm the effect of erlotinib to suppress EGFR activation and signaling through downstream pathways, we performed Western blot analysis of EGFR, Akt, and mTOR phosphorylation in the presence of exogenous EGF (Fig. 5B). EGF treatment stimulated phosphorylation of EGFR and Akt in both control and shLKB1 cells. EGFR phosphorylation was effectively reduced by erlotinib treatment in both cell types, but only shLKB1 Calu-6 cells exhibited reduced phosphorylation of mTOR in response to erlotinib. Therefore, we hypothesized that phosphorylation of AMPK $\alpha$ in LKB1-deficient cells might confer sensitivity to erlotinib through inhibition of mTOR signaling. As shown in Fig. 5C, erlotinib induced phosphorylation of AMPK $\alpha$ in both control and shLKB1 Calu-6 cells after $24 \mathrm{~h}$, but shLKB1 cells exhibited complete disruption of mTOR pathway signaling following erlotinib treatment. In particular, AMPK $\alpha$ can directly phosphorylate TSC2 at Ser 1387, which is involved in inhibition of mTOR signaling. shLKB1 Calu-6 cells exhibited enhanced phosphorylation of TSC2 (Ser1387) by erlotinib treatment in comparison to control cells. Furthermore, 
erlotinib decreased phosphorylation of the 70-kDa ribosomal protein S6 kinase (p70S6K1) and 4EBP1, both downstream effectors of mTOR signaling. Erlotinib treatment reduced phosphorylation of Akt in all cells, but phosphorylated Erk was not changed. These results indicate that erlotinib induces phosphorylation of AMPK $\alpha$ in both control and LKB1-deficient cells, but its effect to block mTOR pathway signaling is enhanced in LKB1-deficient cells, resulting in repression of protein synthesis and inhibition of cell growth.

We then asked whether an alternative kinase other than LKB1 could be responsible for erlotinib-induced activation of AMPK $\alpha$ in LKB1-deficient cells. In a previous study, it was shown that AMPK $\alpha$ can become phosphorylated in response to energy stress, even in the absence of functional LKB1, through the action of calcium/calmodulin-dependent protein kinase kinase 2 $(\mathrm{CaMKK} \beta)(36)$. To elucidate the mechanism by which erlotinib treatment can induce phosphorylation of AMPK $\alpha$ in LKB1-deficient cells, we used STO-609, a CaMKK $\beta$ inhibitor, and BAPTA-AM, an intracellular calcium chelator. After $24 \mathrm{~h}$ of treatment, both STO-609 and BAPTAAM were able to inhibit erlotinib-induced AMPK phosphorylation in shLKB1 Calu-6 cells (Fig. 5D). This blocked erlotinib's ability to reduce mTOR activation and induce apoptosis in shLKB1 Calu-6 cells. These results indicate that AMPK $\alpha$ phosphorylation can be stimulated by erlotinib-induced changes in intracellular ADP/ATP levels through CAMKK $\beta$ activity, even in the absence of functional LKB1. This provides a mechanistic explanation for erlotinib's ability to activate AMPK $\alpha$ and suppress mTOR signaling in LKB1-deficient cells.

\section{Erlotinib reduces tumor volume in shLKB1 Calu-6 tumor xenografts}

Finally, we investigated the in vivo antitumor activity of erlotinib in nude mice bearing control and shLKB1 Calu-6 subcutaneous tumors. As expected, mice bearing shLKB1 Calu-6 tumor xenografts showed the highest tumor volumes (Fig. 6A, left panel). Mean tumor volume in mice bearing shLKB1 tumors was significantly reduced at 2 and 3 weeks of erlotinib treatment, as compared with control-treated mice (Fig. 6A, right panel). No toxic effects (such as severe reduction in body weight) were observed in erlotinib-treated groups. We performed histological analyses of tumor samples by $H \& E$ immunohistochemical staining and found clear signs of large necrotic areas in shLKB1 tumor xenografts treated with erlotinib, but not in control xenografts (Fig. 6B). We also collected tissue samples from tumors to determine the in vivo effects of erlotinib treatment on activation of AMPK $\alpha$, mTOR, p70S6K, and cleaved PARP (Fig. 6C). We first detected levels of LKB1 expression in control and shLKB1 Calu-6 tumor tissues from mice. As shown by Western blotting, levels of LKB1 protein were highly reduced in shLKB1 Calu-6 xenograft tumors as compared to control tumors. Consistent with in vitro results, erlotinib treatment enhanced AMPK phosphorylation, inhibited mTOR-related downstream signaling proteins, and increased PARP cleavage in shLKB1 Calu-6 xenograft tumors only. These results suggest that erlotinib treatment 
significantly reduced tumor growth in shLKB1 Calu-6 tumor xenografts through inhibition of mTOR signaling and induction of apoptosis.

\section{Discussion}

Although erlotinib exhibits potent anti-cancer activity against human NSCLC tumors with activating mutations in $E G F R$, it also has activity in some patients with wild-type $E G F R$. It has been unclear what molecular mechanisms drive this sensitivity and, furthermore, how to identify wild-type $E G F R$ tumors that might be susceptible to erlotinib treatment. In the present study, we found that NSCLC cells harboring wild-type EGFR show varying sensitivity to erlotinib treatment that is dependent on $L K B 1$ status. We demonstrated that erlotinib suppressed viability and enhanced apoptosis of LKB1-deficient NSCLC cells both in vitro and in vivo. Another study recently reported that glioblastoma cells with wild-type EGFR exhibited sensitivity to erlotinib treatment that was associated with inhibition of autophagy but was independent of caspase activity (37). Consistent with this report, our findings indicate that erlotinib can be beneficial even in tumors that are not EGFR mutated. However, we show that apoptosis is elevated in erlotinib-treated cells and that the inhibitory effects of erlotinib are enhanced in tumors that have dysregulated LKB1-AMPK pathway signaling. Because this signaling pathway normally serves to maintain mitochondrial function and bioenergetic homeostasis in response to metabolic stress, we hypothesized that LKB1-deficient cells may be compromised in their ability to cope with metabolic alterations induced by erlotinib treatment.

To further examine the mechanism of erlotinib-induced apoptosis in LKB1-deficient NSCLC cells, we analyzed changes in glycolytic metabolism and mitochondrial function resulting from LKB1 knockdown. Interestingly, we observed that glucose consumption by Calu- 6 cells increased by more than $50 \%$ in response to LKB1 knockdown, which correlated with increased growth and colony formation (Fig. 2C). We also observed substantial increases in OCR (Fig. 3B) and $\Delta \psi_{\mathrm{m}}$ (Fig. 4A) after expression of shLKB1, consistent with a recent gene expression study that profiled over 600 patient tumors and reported upregulation of OXPHOS and mitochondria-associated genes in samples classified as LKB1-deficient (23). Faubert et al. (38) previously reported simultaneous increases in both glucose uptake and lactate production in response to complete $L K B 1$ knockout in transgenic mouse embryonic fibroblasts (MEFs). They also noted enhanced flux of both glucose and glutamine into the TCA cycle of LKB1 mutant A549 cells in comparison to cells expressing wild-type LKB1, based on isotope labeling experiments with ${ }^{13} \mathrm{C}$-glucose and ${ }^{13} \mathrm{C}$-glutamine tracers. These previous findings are consistent with our own results indicating increased dependence of LKB1-deficient cells on mitochondrial metabolism to support enhanced cell proliferation. 
Despite previous reports that LKB1 loss can lead to accumulation of dysfunctional mitochondria as a result of altered mitophagy (39), we observed only slight increases in basal ADP/ATP ratio and ROS levels following expression of shLKB1 in Calu-6 cells (Fig. 3C and 4B). However, the effect of LKB1 depletion to alter mitochondrial function became readily apparent when shLKB1 cells were challenged with erlotinib. Substantial increases in ADP/ATP and ROS levels were observed, which were accompanied by depletion of $\Delta \psi_{\mathrm{m}}$ (Fig. 4A). In contrast, control cells were able to maintain normal ADP/ATP, ROS, and $\Delta \psi_{\mathrm{m}}$ levels in response to the same concentration of erlotinib (Fig. 3C, 4A, 4B). Taken together, these data provide a clear indication that LKB1 is required for maintenance of mitochondrial function in the presence of erlotinib treatment. As a result, LKB1deficient cells cannot effectively restore ATP levels or prevent ROS accumulation in response to erlotinib, resulting in selective growth inhibition (Fig. 2) and apoptosis activation (Fig. 4C and 4D). This is consistent with a previous study that reported increased sensitivity of LKB1-deficient breast cancer cells to undergo apoptosis in response to direct metabolic perturbations, such as glucose depletion (40). Our results are also consistent with another previous study that showed AMPKdeficient cells are unable to maintain sufficient NADPH levels needed to neutralize mitochondrial ROS during metabolic stress (41), a mechanism which may further contribute to the enhanced erlotinib sensitivity we observed in LKB1-deficient cells.

Our observations of increased basal mitochondrial activity in shLKB1 Calu-6 cells are partially at odds with a prior study in A549 cells, which are natively LKB1-deficient. This study showed that re-expressing wild-type LKB1 in A549 cells led to enhancements of basal OCR and $\Delta \psi_{\mathrm{m}}$, implying reduced mitochondrial function in the parental, LKB1-deficient cells (39). Despite this, the parental line was more sensitive to mitochondrial inhibition by phenformin, which selectively induced ROS accumulation and apoptosis in a manner similar to our own observations with erlotinib treatment. In contrast, a more recent study by Faubert et al. (38) showed no change in OCR after re-expressing LKB1 in A549 cells and a reduction in OCR after re-expressing LKB1 in A427 cells, which are also natively LKB1-deficient. Therefore, there is some variability in the prior literature concerning the effects that manipulating LKB1 expression has on markers of mitochondrial activity. It is possible that differences in the basal response to changes in LKB1 expression could be attributable to the fact that natively LKB1-deficient cells have adapted to loss of LKB1 over many generations, and some may have reduced their dependence on mitochondrial metabolism as a way to prevent toxic ROS accumulation. Indeed, Faubert et al. (38) showed that HIF1 $\alpha$ expression can become increased in LKB1-deficient cells due to ROS accumulation, and elevated HIF1 $\alpha$ is at least partially responsible for enhanced lactate production by these cells. The fact that we did not observe an increase in basal ROS in shLKB1 Calu-6 cells may explain why these cells continue to rely on mitochondrial OXPHOS. However, when challenged with erlotinib these cells cannot maintain normal 
mitochondrial function and they are apparently unable to readily increase glycolytic metabolism in order to restore energy homeostasis, control ROS levels, and avoid apoptosis activation.

Another unexpected outcome of our study was that LKB1-deficient cells were still able to increase phosphorylation of AMPK $\alpha$ in response to erlotinib treatment. This effect was inhibited by co-treatment with either STO-609 or BAPTA-AM (Fig. 5D), indicating involvement of CaMKK $\beta$ as an alternative kinase that contributes to erlotinib-induced AMPK phosphorylation. The increase in $\mathrm{ADP} / \mathrm{ATP}$ ratio we observed was therefore sufficient to activate AMPK even when LKB1 was depleted, possibly by a CaMKK $\beta$-dependent mechanism. Consistent with this hypothesis, gefitinib has been reported to increase cytosolic and mitochondrial calcium levels of $\mathrm{Bcl}$-2-overexpressing cells (42). Moreover, erlotinib-mediated AMPK $\alpha$ activation led to inhibition of mTOR phosphorylation in LKB1-deficient cells, which correlated with inhibition of phosphorylated p70S6K and 4EBP-1 (Fig. 5C) as major downstream effectors of the mTOR signaling pathway. These data are consistent with the known role of AMPK to suppress mTOR signaling in response to low ATP levels, leading to inhibition of protein synthesis and proliferation of cancer cells $(10,43)$. Previous studies suggested that loss of LKB1 induced increased cell size and mass due to failure of AMPK to inhibit mTOR signaling $(31,32)$. In contrast, we found that only LKB1-deficient cells effectively suppressed mTOR signaling and induced PARP cleavage in response to erlotinib treatments, both in vitro and in vivo. This led to a significant decrease in growth of LKB1-deficient xenograft tumors. It is likely that mitochondrial dysfunction associated with LKB1 deficiency sensitizes cells to erlotinib treatment by enhancing changes in ADP/ATP and ROS levels, which subsequently activate AMPK and suppress mTOR signaling.

In conclusion, we demonstrate for the first time that erlotinib induces apoptosis in $E G F R$ wild-type NSCLC cells by a mechanism that is enhanced by LKB1 loss of function. Although LKB1deficient cells relied more heavily on mitochondrial OXPHOS for energy production under basal conditions, they could not properly respond to metabolic stress induced by erlotinib treatment in order to maintain energetic homeostasis. These metabolic alterations were associated with more rapid AMPK $\alpha$ phosphorylation and inhibition of mTOR signaling in LKB1-deficient cells, resulting in increased sensitivity to erlotinib treatment. Taken together, our findings provide a rationale for erlotinib treatment of LKB1-deficient NSCLC tumors, even in the absence of EGFR activating mutations. These tumors may constitute a distinct NSCLC molecular subtype with enhanced erlotinib sensitivity, which motivates the development of novel diagnostic approaches (e.g., (23)) capable of identifying LKB1-deficient patient tumors that are potentially susceptible to erlotinib treatment.

\section{Acknowledgments}


This work was supported by NIH P50 CA090949, DOD W81XWH-12-1-0383, NSF

CBET-1105991, and an award from Uniting Against Lung Cancer. The authors thank Dr. Changki

Lee for his assistance with the animal experiments.

\section{References}

1. Jemal A, Siegel R, Xu J, Ward E. Cancer Statistics, 2010. CA: A Cancer Journal for Clinicians. 2010;60:277-300.

2. Rapp E, Pater JL, Willan A, Cormier Y, Murray N, Evans WK, et al. Chemotherapy can prolong survival in patients with advanced non-small-cell lung cancer--report of a Canadian multicenter randomized trial. 1988.

3. West L, Vidwans SJ, Campbell NP, Shrager J, Simon GR, Bueno R, et al. A novel classification of lung cancer into molecular subtypes. PLoS One. 2012;7:e31906.

4. Kwak EL, Bang YJ, Camidge DR, Shaw AT, Solomon B, Maki RG, et al. Anaplastic lymphoma kinase inhibition in non-small-cell lung cancer. The New England journal of medicine. 2010;363:1693-703.

5. Tsao MS, Sakurada A, Cutz JC, Zhu CQ, Kamel-Reid S, Squire J, et al. Erlotinib in lung cancer - molecular and clinical predictors of outcome. The New England journal of medicine. 2005;353:133-44.

6. Shackelford DB, Shaw RJ. The LKB1-AMPK pathway: metabolism and growth control in tumour suppression. Nat Rev Cancer. 2009;9:563-75.

7. Sanchez-Cespedes M, Parrella P, Esteller M, Nomoto S, Trink B, Engles JM, et al. Inactivation of LKB1/STK11 Is a Common Event in Adenocarcinomas of the Lung. 2002.

8. Ji H, Ramsey MR, Hayes DN, Fan C, McNamara K, Kozlowski P, et al. LKB1 modulates lung cancer differentiation and metastasis. Nature. 2007;448:807-10.

9. Hardie DG, Ross FA, Hawley SA. AMPK: a nutrient and energy sensor that maintains energy homeostasis. Nat Rev Mol Cell Biol. 2012;13:251-62.

10. Mihaylova MM, Shaw RJ. The AMPK signalling pathway coordinates cell growth, autophagy and metabolism. Nat Cell Biol. 2011;13:1016-23.

11. Carretero J, Medina PP, Blanco R, Smit L, Tang M, Roncador G, et al. Dysfunctional AMPK activity, signalling through mTOR and survival in response to energetic stress in LKB1-deficient lung cancer. Oncogene. 2007;26:1616-25.

12. Shackelford David B, Abt E, Gerken L, Vasquez Debbie S, Seki A, Leblanc M, et al. LKB1 Inactivation Dictates Therapeutic Response of Non-Small Cell Lung Cancer to the Metabolism Drug Phenformin. Cancer Cell. 2013;23:143-58.

13. Faber AC, Li D, Song Y, Liang M-C, Yeap BY, Bronson RT, et al. Differential induction of apoptosis in HER2 and EGFR addicted cancers following PI3K inhibition. Proceedings of the National Academy of Sciences. 2009;106:19503-8.

14. Pao W, Miller V, Zakowski M, Doherty J, Politi K, Sarkaria I, et al. EGF receptor gene mutations are common in lung cancers from "never smokers" and are associated with sensitivity of tumors to gefitinib and erlotinib. Proc Natl Acad Sci U S A. 2004;101:13306-11.

15. Sharma SV, Bell DW, Settleman J, Haber DA. Epidermal growth factor receptor mutations in lung cancer. Nat Rev Cancer. 2007;7:169-81.

16. Shepherd FA, Rodrigues Pereira J, Ciuleanu T, Tan EH, Hirsh V, Thongprasert S, et al. Erlotinib in Previously Treated Non-Small-Cell Lung Cancer. New England Journal of Medicine. 2005;353:123-32.

17. Cappuzzo F, Ciuleanu T, Stelmakh L, Cicenas S, Szczésna A, Juhász E, et al. Erlotinib as maintenance treatment in advanced non-small-cell lung cancer: a multicentre, randomised, placebocontrolled phase 3 study. The Lancet Oncology. 2010;11:521-9. 
18. Garassino MC, Marsoni S, Floriani I. Testing Epidermal Growth Factor Receptor Mutations in Patients With Non-Small-Cell Lung Cancer to Choose Chemotherapy: The Other Side of the Coin. 2011.

19. Deng J, Shimamura T, Perera S, Carlson NE, Cai D, Shapiro GI, et al. Proapoptotic BH3Only BCL-2 Family Protein BIM Connects Death Signaling from Epidermal Growth Factor Receptor Inhibition to the Mitochondrion. 2007.

20. Ling Y-H, Lin R, Perez-Soler R. Erlotinib Induces Mitochondrial-Mediated Apoptosis in Human H3255 Non-Small-Cell Lung Cancer Cells with Epidermal Growth Factor ReceptorL858R Mutation through Mitochondrial Oxidative Phosphorylation-Dependent Activation of BAX and BAK. 2008.

21. Nakada D, Saunders TL, Morrison SJ. Lkb1 regulates cell cycle and energy metabolism in haematopoietic stem cells. Nature. 2010;468:653-8.

22. Matsumoto S, Iwakawa R, Takahashi K, Kohno T, Nakanishi Y, Matsuno Y, et al. Prevalence and specificity of LKB1 genetic alterations in lung cancers. Oncogene. 2007;26:5911-8.

23. Kaufman JM, Amann JM, Park K, Arasada RR, Li H, Shyr Y, et al. LKB1 Loss Induces Characteristic Patterns of Gene Expression in Human Tumors Associated with NRF2 Activation and Attenuation of PI3K-AKT. Journal of Thoracic Oncology. 2014;9.

24. Murphy TA, Young JD. ETA: Robust software for determination of cell specific rates from extracellular time courses. Biotechnology and Bioengineering. 2013;110:1748-58.

25. Whang YM, Kim YH, Kim JS, Yoo YD. RASSF1A Suppresses the c-Jun-NH2-Kinase Pathway and Inhibits Cell Cycle Progression. 2005.

26. Park SI, Lee C, Sadler WD, Koh AJ, Jones J, Seo JW, et al. Parathyroid Hormone-Related Protein Drives a CD11b+Gr1+ Cell-Mediated Positive Feedback Loop to Support Prostate Cancer Growth. 2013.

27. Van Schaeybroeck S, Kyula J, Kelly DM, Karaiskou-McCaul A, Stokesberry SA, Van

Cutsem E, et al. Chemotherapy-induced epidermal growth factor receptor activation determines response to combined gefitinib/chemotherapy treatment in non-small cell lung cancer cells.

Molecular Cancer Therapeutics. 2006;5:1154-65.

28. Tracy S, Mukohara T, Hansen M, Meyerson M, Johnson BE, Jänne PA. Gefitinib Induces Apoptosis in the EGFRL858R Non-Small-Cell Lung Cancer Cell Line H3255. Cancer Research. 2004;64:7241-4.

29. Onozato R, Kosaka T, Achiwa H, Kuwano H, Takahashi T, Yatabe Y, et al. LKB1 gene mutations in Japanese lung cancer patients. Cancer Science. 2007;98:1747-51.

30. Liu Y, Marks K, Cowley GS, Carretero J, Liu Q, Nieland TJF, et al. Metabolic and Functional Genomic Studies Identify Deoxythymidylate Kinase as a Target in LKB1-Mutant Lung Cancer. Cancer Discovery. 2013;3:870-9.

31. Mahoney CL, Choudhury B, Davies H, Edkins S, Greenman C, Haaften G, et al. LKB1/KRAS mutant lung cancers constitute a genetic subset of NSCLC with increased sensitivity to MAPK and mTOR signalling inhibition. Br J Cancer2009. p. 370-5.

32. Woods A, Johnstone SR, Dickerson K, Leiper FC, Fryer LGD, Neumann D, et al. LKB1 Is the Upstream Kinase in the AMP-Activated Protein Kinase Cascade. Current Biology. 2003;13:20048.

33. Shaw RJ, Kosmatka M, Bardeesy N, Hurley RL, Witters LA, DePinho RA, et al. The tumor suppressor LKB1 kinase directly activates AMP-activated kinase and regulates apoptosis in response to energy stress. Proceedings of the National Academy of Sciences of the United States of America. 2004;101:3329-35.

34. M S, P G, B H, M S, D C. Investigating the mechanism for AMP activation of the AMPactivated protein kinase cascade. 2007.

35. Faubert B, Vincent EE, Griss T, Samborska B, Izreig S, Svensson RU, et al. Loss of the tumor suppressor LKB1 promotes metabolic reprogramming of cancer cells via HIF-1 $\alpha$. Proceedings of the National Academy of Sciences. 2014;111:2554-9.

36. Hurley RL, Anderson KA, Franzone JM, Kemp BE, Means AR, Witters LA. The $\mathrm{Ca} 2+/$ Calmodulin-dependent Protein Kinase Kinases Are AMP-activated Protein Kinase Kinases. Journal of Biological Chemistry. 2005;280:29060-6. 
37. Eimer S, Belaud-Rotureau M-A, Airiau K, Jeanneteau M, Laharanne E, Véron N, et al. Autophagy inhibition cooperates with erlotinib to induce glioblastoma cell death. Cancer Biology \& Therapy. 2011;11:1017-27.

38. Faubert B, Vincent EE, Griss T, Samborska B, Izreig S, Svensson RU, et al. Loss of the tumor suppressor LKB1 promotes metabolic reprogramming of cancer cells via HIF-1alpha. Proc Natl Acad Sci U S A. 2014;111:2554-9.

39. Shackelford DB, Abt E, Gerken L, Vasquez DS, Seki A, Leblanc M, et al. LKB1 inactivation dictates therapeutic response of non-small cell lung cancer to the metabolism drug phenformin. Cancer Cell. 2013;23:143-58.

40. Dupuy F, Griss T, Blagih J, Bridon G, Avizonis D, Ling C, et al. LKB1 is a central regulator of tumor initiation and pro-growth metabolism in ErbB2-mediated breast cancer. Cancer Metab. $2013 ; 1: 18$.

41. Jeon SM, Chandel NS, Hay N. AMPK regulates NADPH homeostasis to promote tumour cell survival during energy stress. Nature. 2012;485:661-5.

42. Zannetti A, Iommelli F, Fonti R, Papaccioli A, Sommella J, Lettieri A, et al. Gefitinib Induction of In vivo Detectable Signals by Bcl-2/Bcl-xL Modulation of Inositol Trisphosphate Receptor Type 3. Clinical Cancer Research. 2008;14:5209-19.

43. Shaw RJ, Bardeesy N, Manning BD, Lopez L, Kosmatka M, DePinho RA, et al. The LKB1 tumor suppressor negatively regulates mTOR signaling. Cancer Cell. 2004;6:91-9. 


\section{Figure Captions}

Figure 1. Erlotinib and inhibitors of PI3K-mTOR signaling selectively inhibit growth of LKB1deficient NSCLC cells. A, Levels of LKB1 expression and EGFR phosphorylation in our panel of NSCLC cells. Actin was used as a loading control. B, Effect of treatment with erlotinib, LY294002, or rapamycin on viability of NSCLC cells. Cells were seeded in 96-well plates. After $24 \mathrm{~h}$, cells were treated with indicated concentrations of erlotinib, LY294002, or rapamycin for $48 \mathrm{~h}$. Cell viability was determined using MTT assay. *; $p<0.005$, **; $p<0.00001$, untreated/erlotinib, *; $p<0.0001$, untreated/LY294002 or untreated/rapamycin. Data are mean \pm SEM $(n=6)$. C, Effects of treatment with erlotinib, LY294002, or rapamycin on colony-forming ability of NSCLC cells. After $72 \mathrm{~h}$ treatment with erlotinib, LY294002, or rapamycin, cells were re-plated in 12-well plates at low density. Colonies were counted after 2 weeks of growth. *; $p<0.0001$, untreated/erlotinib, untreated/LY294002, or untreated/rapamycin. Data are mean $\pm \operatorname{SEM~(n=3).~}$

Figure 2. Erlotinib selectively inhibits growth of LKB1 mutant cells and LKB1 knockdown cells. A, Left panel: Levels of LKB1 expression and LKB1-related downstream proteins in A549 and H460 cells stably expressing empty pBABE vector (vector) or wild-type LKB1 (LKB1). Right panel: Cell viability determined by MTT assay. Cells were treated with indicated concentrations of erlotinib for 48 h. *; $p<0.005,{ }^{* *} ; p<0.0001$, untreated/erlotinib. Data are mean $\pm \operatorname{SEM}(\mathrm{n}=6)$. B, Upper panel: Western blots of whole-cell lysates from LKB1 stable knockdown and non-targeted shRNA control Calu- 6 cells after puromycin selection: control, pLKO.1 control vector; shLKB1\#1 and shLKB1\#2, LKB1 shRNA vectors. Lower panel: Cell viability determined by MTT assay. *; $p<0.0001$, untreated/erlotinib. Data are mean $\pm \operatorname{SEM}(n=6)$. C, Colony-forming ability of LKB1 stable knockdown Calu-6 cells. After $72 \mathrm{~h}$ treatment with $5 \mu \mathrm{M}$ erlotinib, cells were replated in 12-well plates at low density. Colonies were counted after 2 weeks of growth. *; $p<0.001$, untreated/erlotinib. Data are mean $\pm \operatorname{SEM}(n=3)$.

Figure 3. Erlotinib reduces growth rate and selectively inhibits OXPHOS in LKB1-deficient cells. A, Cell growth rate, glucose uptake rate, and lactate production rate of Calu-6 cells. Cells were grown for $72 \mathrm{~h}$ in the presence of treatments. B, Oxygen consumption rate (OCR) of Calu-6 cells. Cells were treated with erlotinib for $24 \mathrm{~h}$. Data are mean $\pm \operatorname{SEM}(\mathrm{n}=4)$. *; $p<0.005$, untreated/erlotinib. C, ADP/ATP ratio of Calu- 6 cells. Cells were treated with $25 \mu \mathrm{M}$ erlotinib for 24 h or $1 \mathrm{mM} \mathrm{H}_{2} \mathrm{O}_{2}$ (positive control) for $1 \mathrm{~h}$ prior to measurement. Data are mean $\pm \mathrm{SEM}$. *; $\mathrm{p}<0.05$, **; $\mathrm{p}<0.005$, untreated/erlotinib.

Figure 4. Erlotinib induces mitochondrial defects and enhanced apoptosis in stable LKB1 knockdown NSCLC cells. A, Upper panel: Western blot analysis of LKB1 expression in H358 cells. 
Mitochondrial potential was measured using JC-1 fluorescence in both H358 (middle panel) and Calu6 cells (bottom panel). Cells were treated with $25 \mu \mathrm{M}$ erlotinib for $24 \mathrm{~h}$. Data are mean \pm SEM. *; $p<0.05$, untreated/erlotinib. B, Mitochondrial ROS accumulation by Calu- 6 cells. Cells were treated with $25 \mu \mathrm{M}$ erlotinib for $24 \mathrm{~h}$ or $20 \mu \mathrm{M}$ antimycin A (positive control) for 40 min prior to staining with MitoSOX Red and analysis by flow cytometry. C, Induction of apoptosis by erlotinib in Calu-6 cells. Control and shLKB1 cells were treated with $25 \mu \mathrm{M}$ erlotinib for $24 \mathrm{~h}$ and cell lysates were then subjected to Western blot analysis using antibody to cleaved PARP. D, Apoptosis was detected with Annexin V staining in control and shLKB1 Calu-6 cells following $48 \mathrm{~h}$ treatment with erlotinib.

Figure 5. Erlotinib-induced activation of AMPKa selectively inhibits mTOR signaling pathway in LKB1-deficient cells. A, Erlotinib activated AMPK $\alpha$ and inhibited ACC in shLKB1 Calu- 6 cells. Following treatment with $0.5 \mu \mathrm{M}$ erlotinib for the indicated time, cell lysates were analyzed by Western blotting. The expression of phosphorylated AMPK $\alpha$ was calculated as the ratio of phosphorylated AMPK $\alpha$ to total AMPK $\alpha$ protein expression using densitometric analysis (left panel). Erlotinib also induced phosphorylated AMPKa in LKB1 mutant H23 and H460 cells (right panel). B, Erlotinib inhibited EGF-stimulated EGFR phosphorylation and mTOR phosphorylation in shLKB1 Calu-6 cells. Cells were treated with $0.5 \mu \mathrm{M}$ erlotinib for $72 \mathrm{~h}$. After serum starvation for $24 \mathrm{~h}$, cells were stimulated by $100 \mathrm{ng} / \mathrm{ml}$ EGF for 10 minutes and cell lysates were then analyzed by Western blotting. C, Erlotinib blocked mTOR pathway signaling and activated AMPK $\alpha$ in shLKB1 Calu-6 cells. After $24 \mathrm{~h}$ of $0.5 \mu \mathrm{M}$ erlotinib treatment, cell lysates were analyzed by Western blotting using specific antibodies for the indicated proteins. Blockade of mTOR activity following erlotinib treatment resulted in decreased phosphorylation of p70S6K and p-4EBP1 in shLKB1 Calu-6 cells. D, Erlotinib-induced pAMPK $\alpha$ phosphorylation was reduced by addition of STO-609 (a CAMKK $\beta$ inhibitor) or BAPTA-AM (an intracellular calcium chelator) in shLKB1 Calu-6 cells. Cells were cotreated with $0.5 \mu \mathrm{M}$ erlotinib and $2.5 \mu \mathrm{M}$ STO-609 or $4 \mu \mathrm{M}$ BAPTA-AM for $24 \mathrm{~h}$ and cell lysates were analyzed by Western blotting. The blots are representative of three independent experiments.

Figure 6. Erlotinib induces a significant reduction of tumor volume in shLKB1 mice. A, Mean tumor volumes for each treatment group of the pLKO.1 control vector (dil, $n=10$, erlo, $n=8$ ) and shLKB1 vector (dil, $n=10$, erlo, $n=10$ ) mice after 1 week of treatment. Female nude mice were injected subcutaneously into the left/right flanks with $5 \times 10^{6}$ control or shLKB1 Calu- 6 cells per mouse mixed 1:1 with 100\% Matrigel. After 7 days, mice were treated with diluent (dil) or erlotinib (erlo) in sodium carboxymethylcellulose and Tween 80 solution o.p. at $100 \mathrm{mg} / \mathrm{kg}$ daily. Body weight was measured twice a week, and drug administration was adjusted accordingly. Tumors were measured twice a week with a caliper. Dots: individual tumor volumes $\left(\mathrm{mm}^{3}\right)$. Horizontal lines: median and inter-quartile ranges. p-values are from linear regression analyses and Mann Whitney $\mathrm{U}$ test. $\mathbf{B}, H \& E$ immunohistochemical staining of sectioned tumor tissues. Original magnification, $\times 20$. 
Scale bars, $100 \mu \mathrm{m}$. C, Erlotinib inhibited mTOR signaling and enhanced cleaved PARP in shLKB1 Calu-6 tumor xenografts. The blots are representative of three independent experiments.

Figure 7. Hypothetical schema of erlotinib-induced cell death in LKB1-deficient cells. LKB1deficient cells exhibit enhanced mitochondrial metabolism as indicated by increased mitochondrial potential and OCR. Erlotinib treatment selectively induces mitochondrial dysfunction in LKB1deficient cells, decreasing mitochondrial potential and OCR while increasing ADP/ATP and ROS. This has the dual effect of inhibiting growth (via activation of AMPK and suppression of mTOR signaling) and activating apoptosis. 
Fig. 1

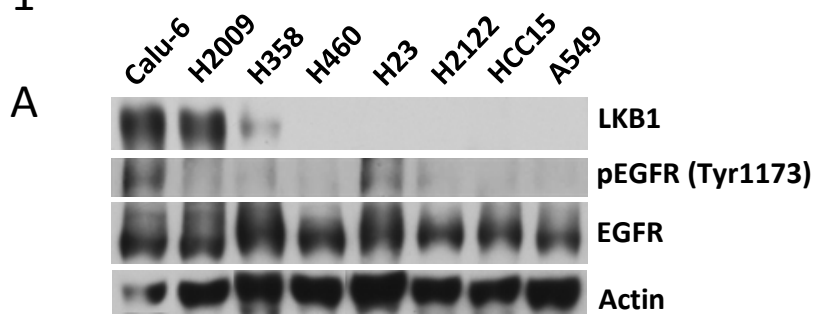

B
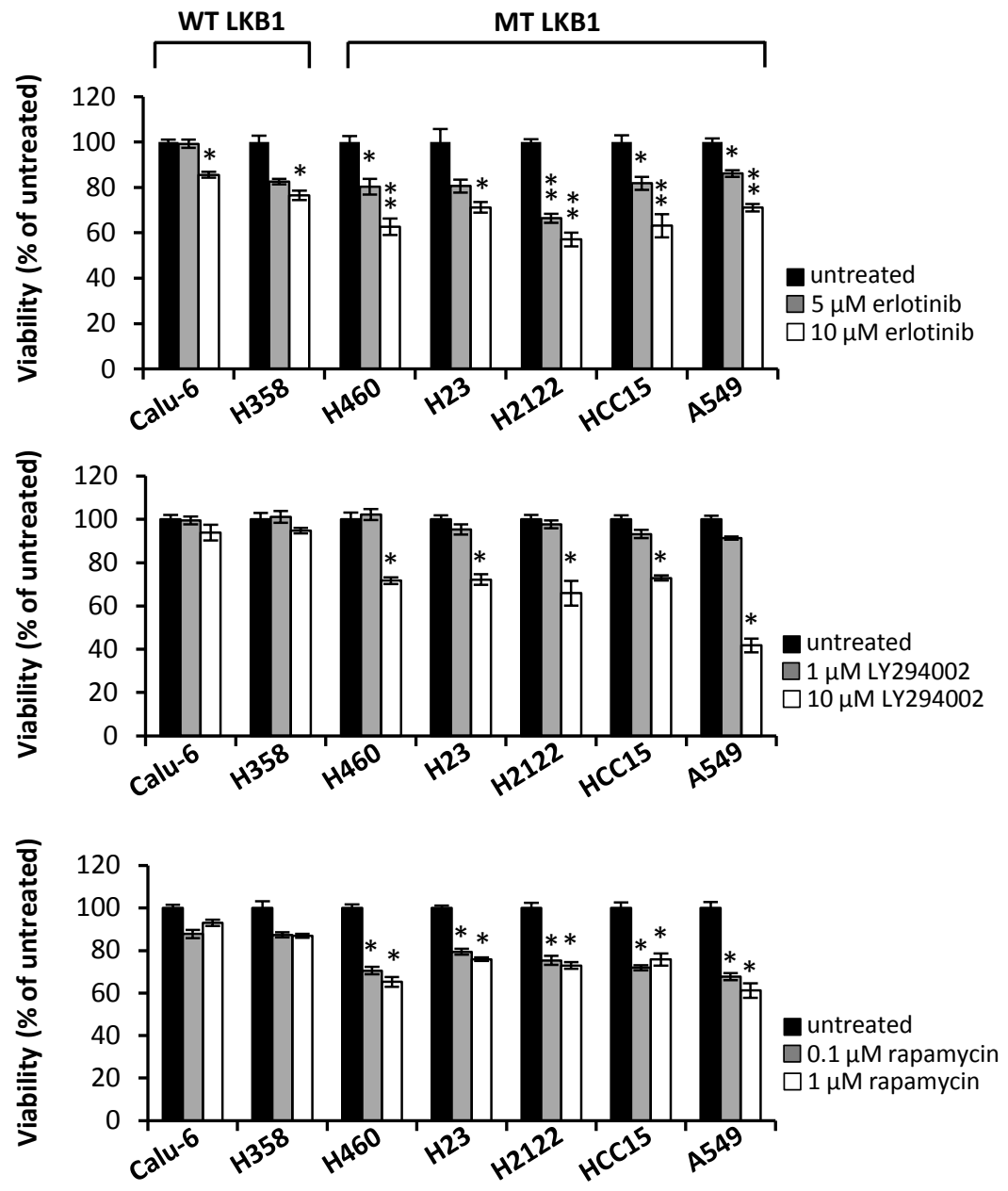

C

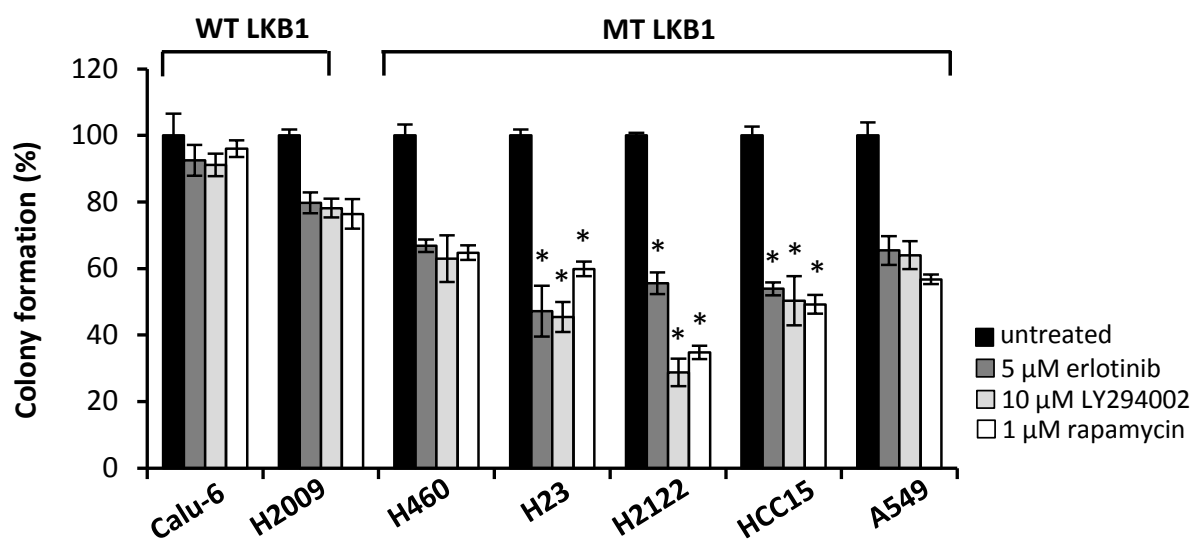


Fig. 2

A
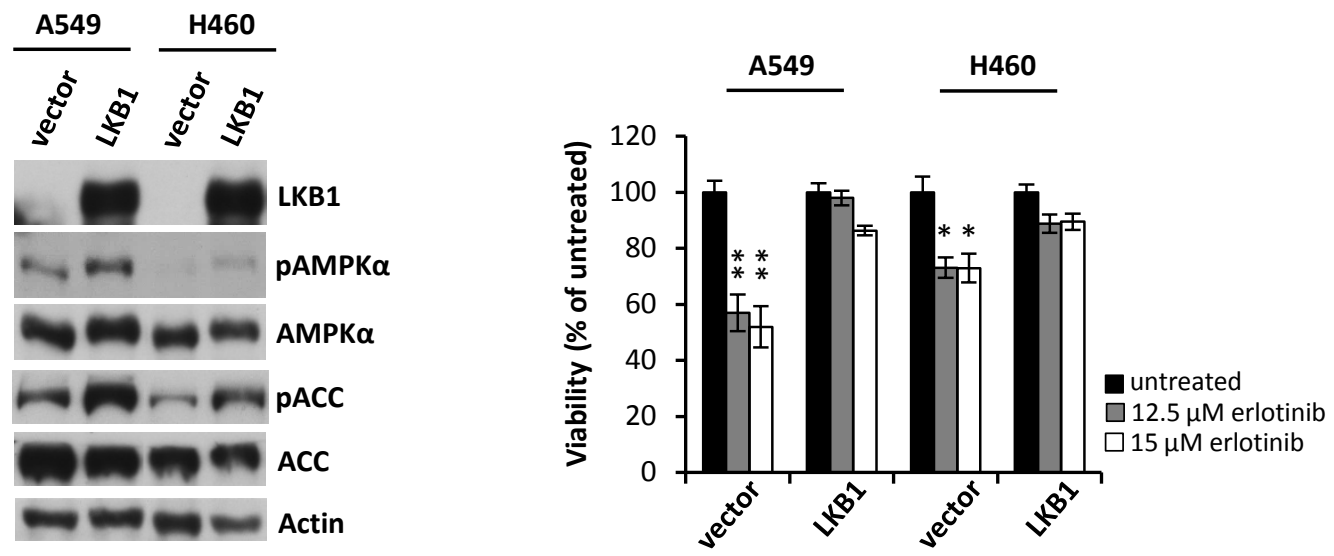

B

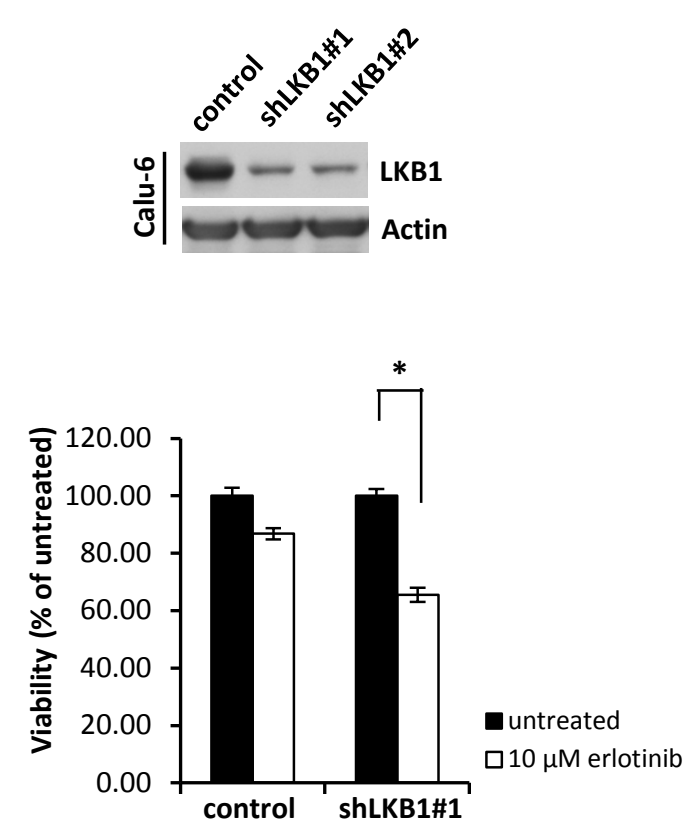

C
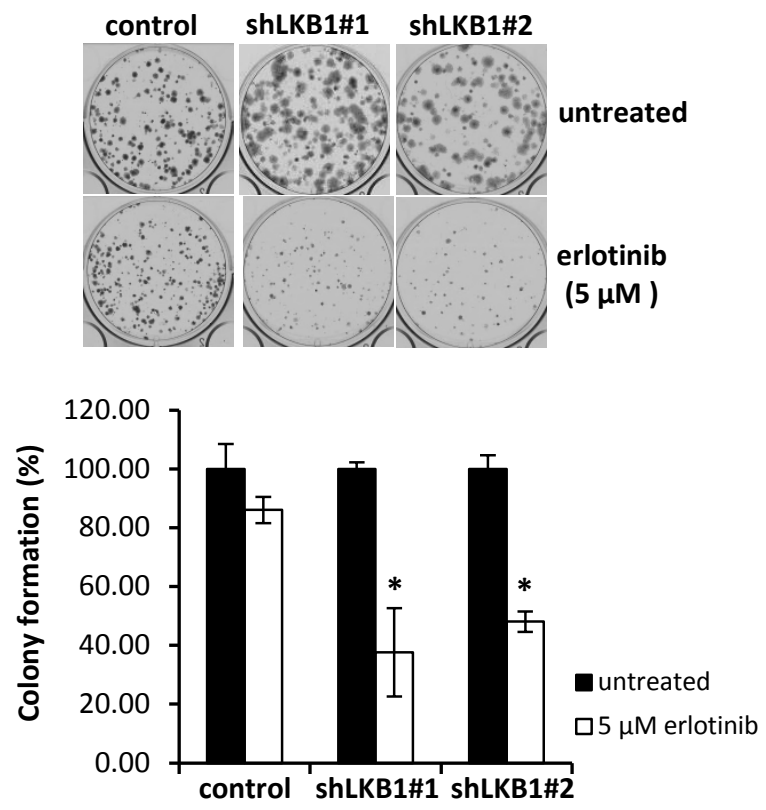
Fig. 3

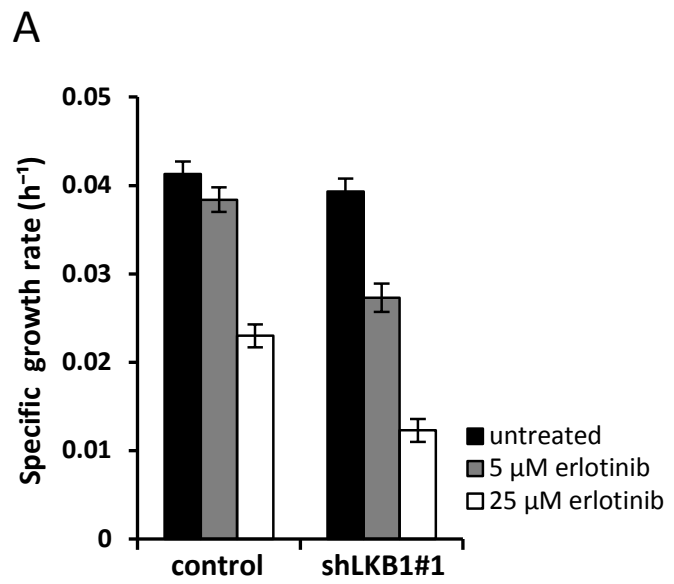

B
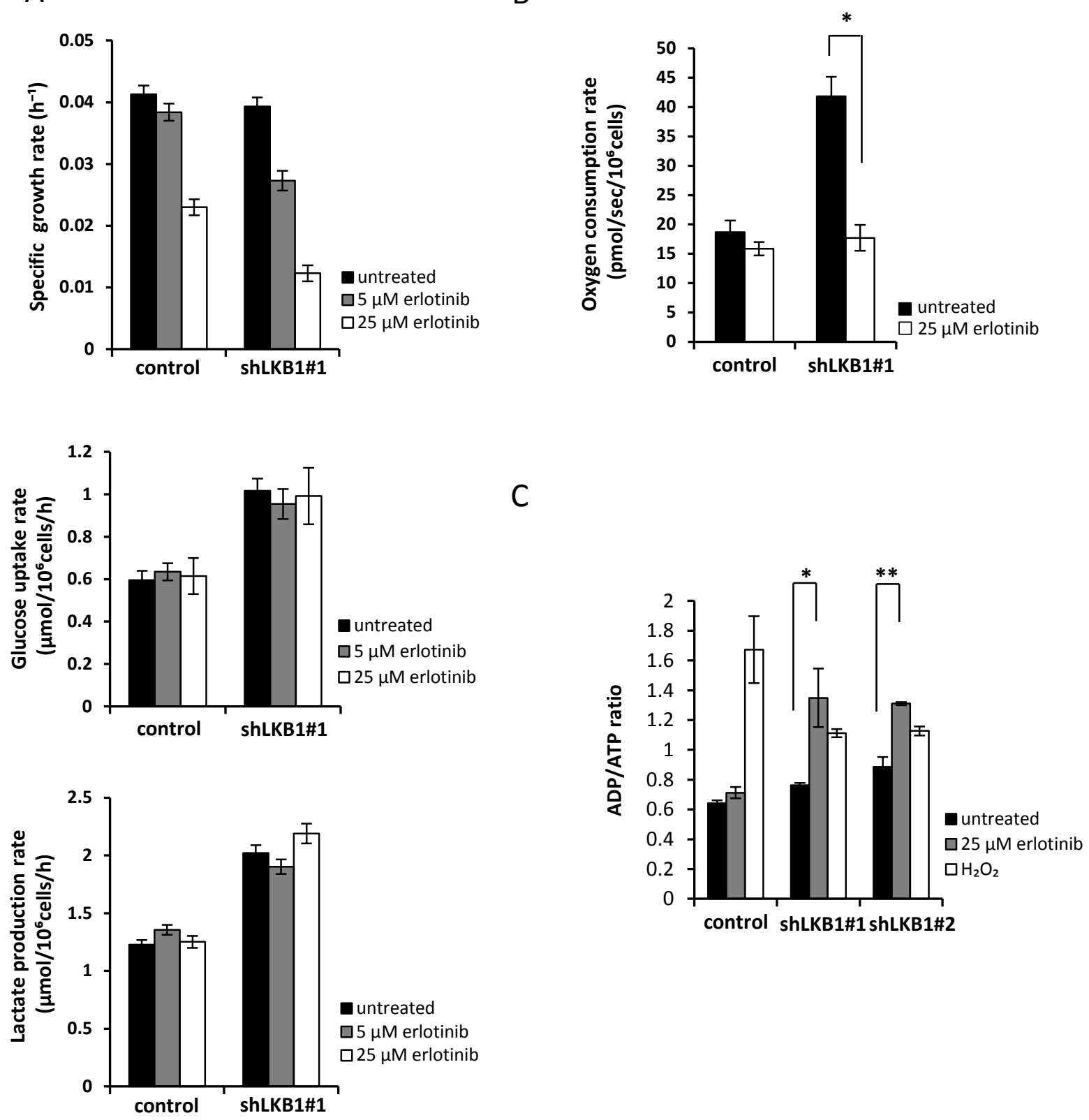
Fig. 4

A
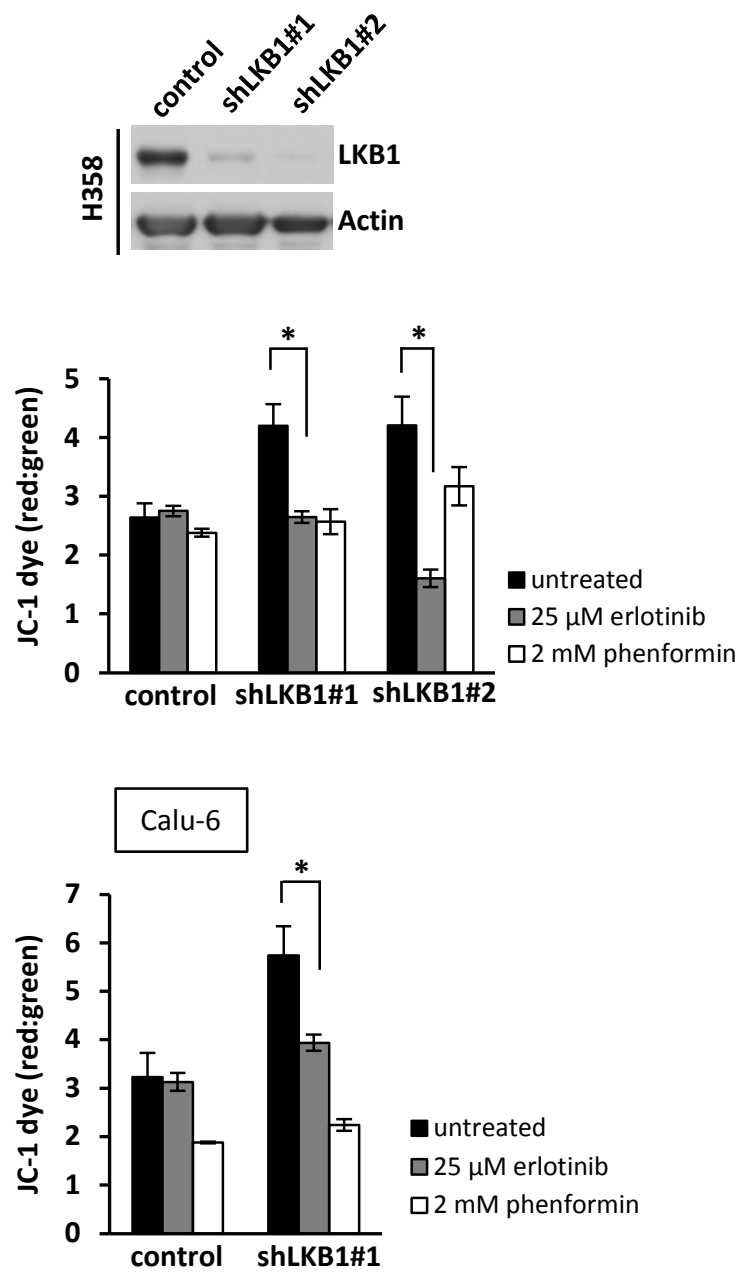

B

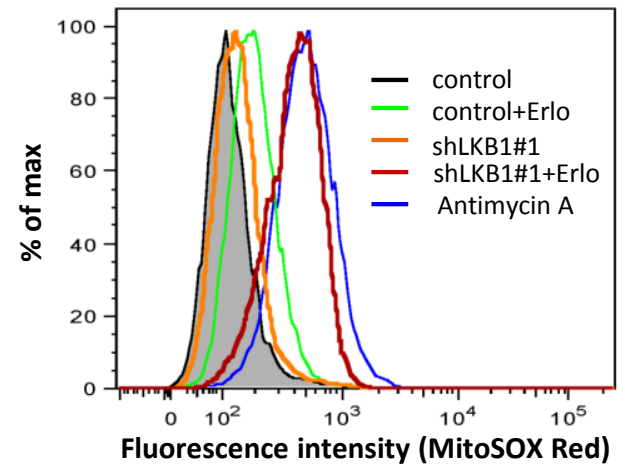

C

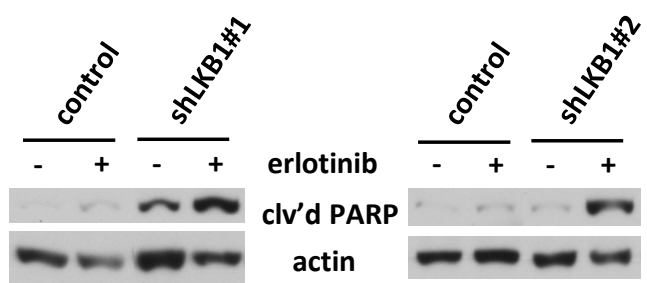

D

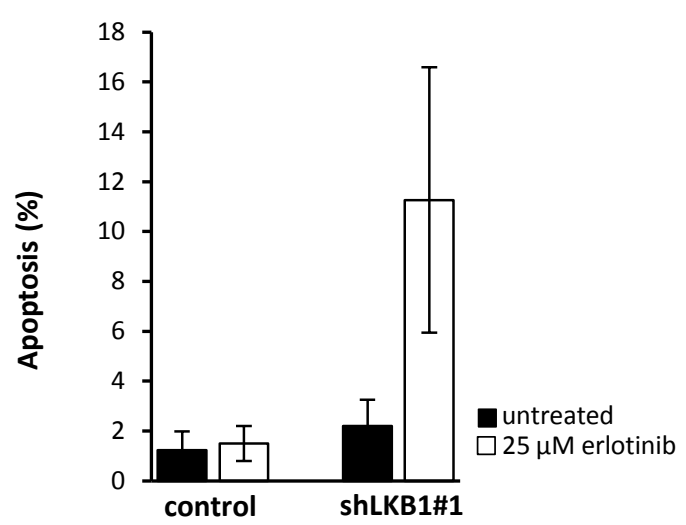


Fig. 5

A

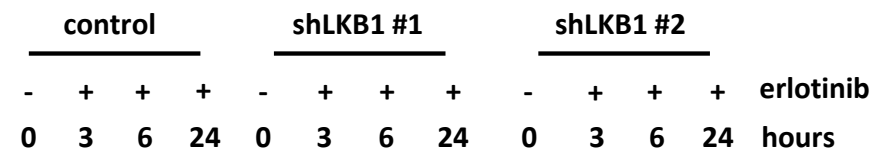

$\begin{array}{lllllllllll}1 & 0.93 & 0.96 & 1.33 & 1.141 .18 & 1.65 & 1.57 & 1.05 & 1.15 & 1.35 & 1.34\end{array}$

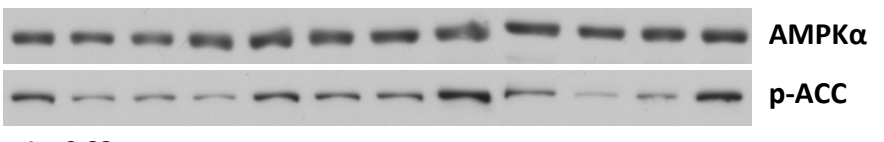

$\begin{array}{llllllllllll}1 & 0.83 & 0.75 & 0.8 & 0.99 & 0.95 & 0.97 & 1.34 & 0.91 & 0.75 & 0.86 & 1.29\end{array}$

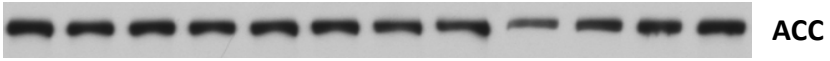

B

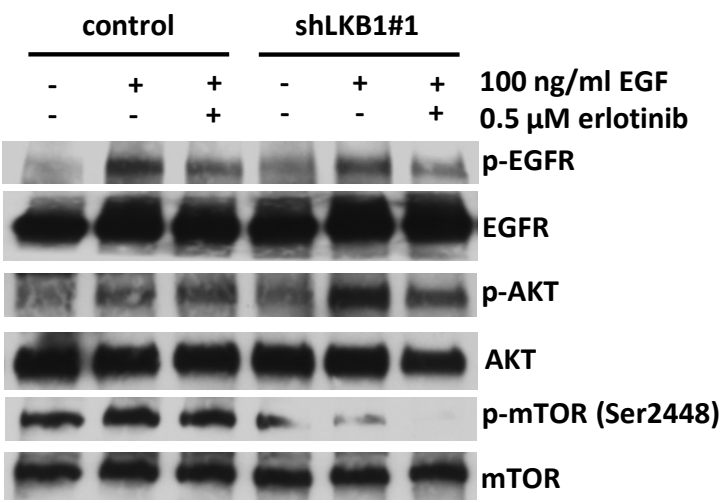

D

\begin{tabular}{ccccccccccccc}
\multicolumn{3}{c}{ control } & \multicolumn{8}{c}{ shLKB1 } \\
\cline { 1 - 1 } & + & - & + & - & + & - & + & - & + & - & + \\
- & - & + & + & - & - & - & - & + & + & - & - \\
- & - & - & - & + & + & - & - & - & - & + & +
\end{tabular}

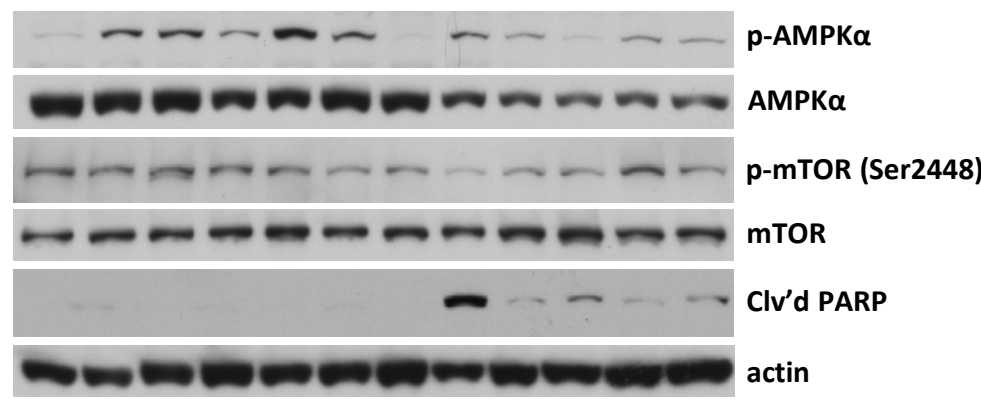

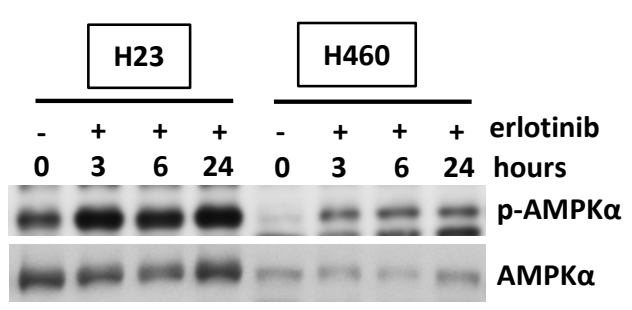

C

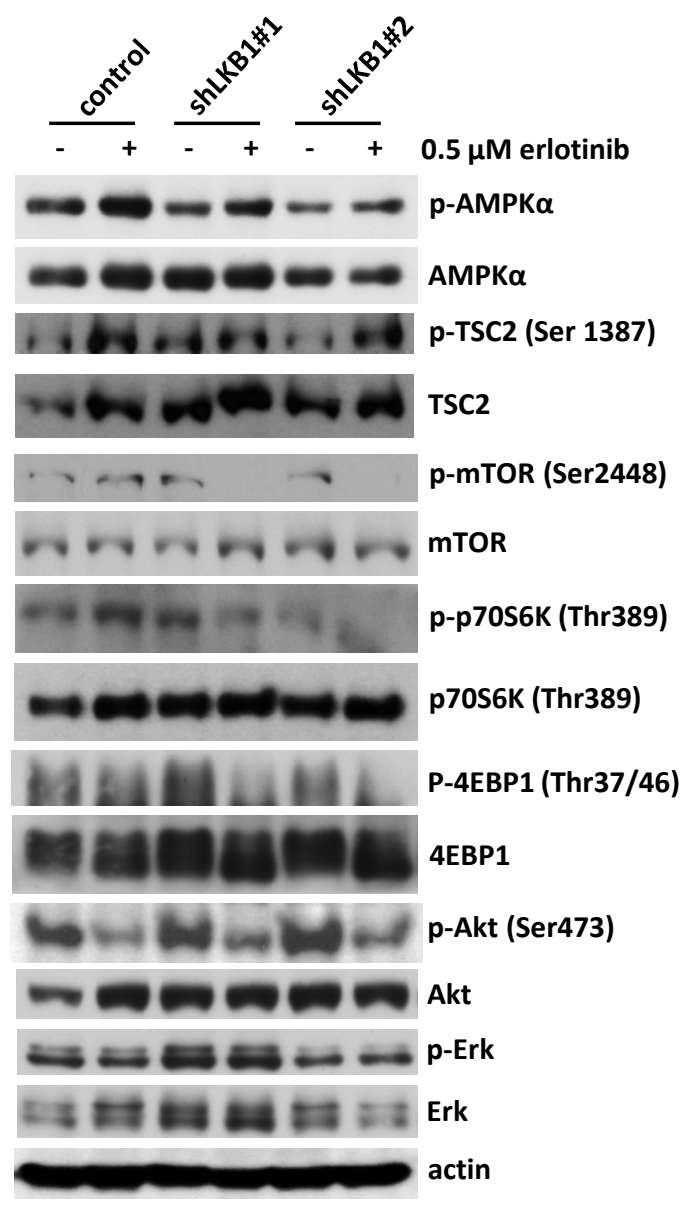


Fig. 6

A

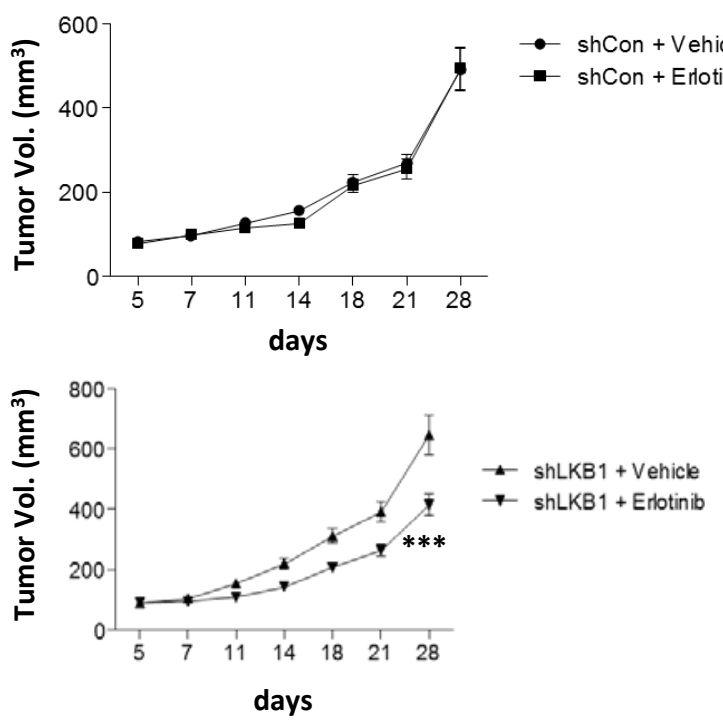

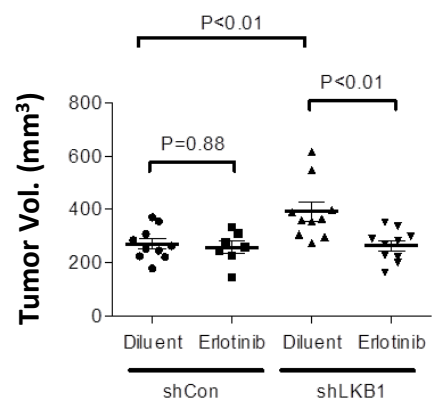

Two weeks of treatment

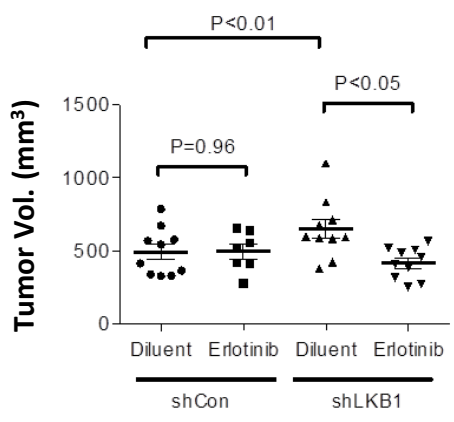

Three weeks of treatment

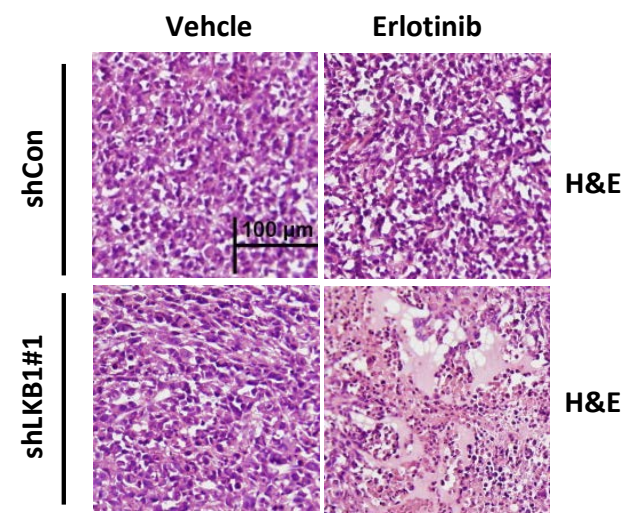

C

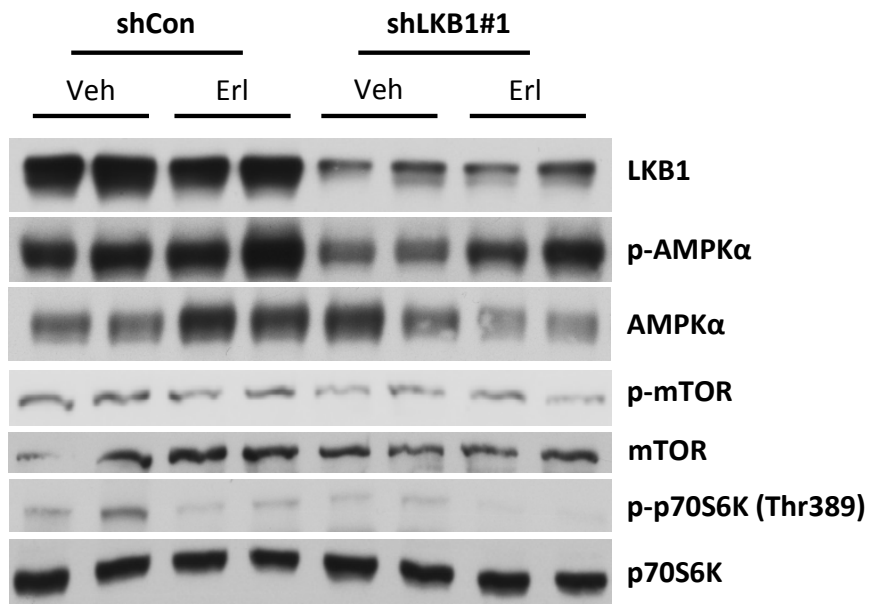

Clv'd PARP

Actin 
Fig. 7

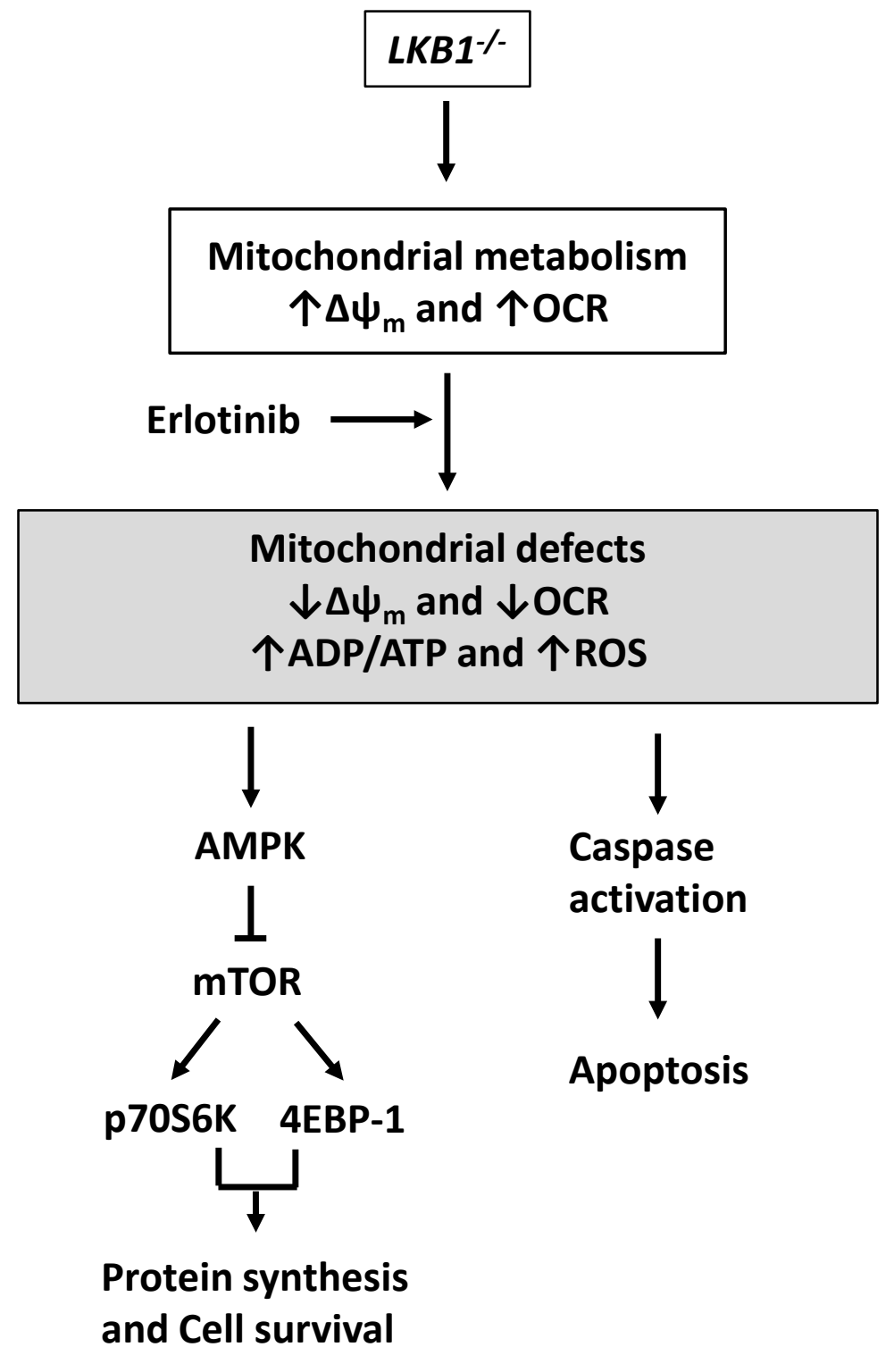

